\title{
Numerical Simulation of Phase Transition Problems with Explicit Interface Tracking
}

\author{
Yijing $\mathrm{Hu}^{\mathrm{a}}$, Qiangqiang Shi ${ }^{\mathrm{a}}$, Valmor F. De Almeida ${ }^{\mathrm{b}}$, Xiaolin $\mathrm{Li}^{\mathrm{a}}$ \\ ${ }^{a}$ Department of Applied Mathematics and Statistics, Stony Brook University, Stony \\ Brook, NY 11794-3600, United States \\ ${ }^{b}$ Oak Ridge National Laboratory, Oak Ridge, TN 37831-6181, United States \\ Telephone: +1-631-488-7758; Email: qqshi@ams.sunysb.edu
}

\begin{abstract}
Phase change is ubiquitous in nature and industrial processes. Started from the Stefan problem, it is a topic with a long history in applied mathematics and sciences and continues to generate outstanding mathematical problems. For instance, the explicit tracking of the Gibbs dividing surface between phases is still a grand challenge. Our work has been motivated by such challenge and here we report on progress made in solving the governing equations of continuum transport in the presence of a moving interface by the front tracking method. The most pressing issue is the accounting of topological changes suffered by the interface between phases wherein break up and/or merge takes place. The underlying physics of topological changes require the incorporation of space-time subscales not at reach at the moment. Therefore we use heuristic geometrical arguments to reconnect phases in space. This heuristic approach provides new insight in various applications and it is extensible to include subscale physics and chemistry in the future. We demonstrate the method on applications such as simulating freezing, melting, dissolution, and precipitation. The later examples also include the coupling of the phase transition solution with the Navier-Stokes equations for the effect of flow convection.
\end{abstract}

Keywords: Front tracking; Phase transition; Precipitation; Dissolution; Freezing; Melting 


\section{Introduction}

Phase transition process is one of the important processes in chemical reaction and physical state transformation. It transforms a thermodynamic system from one phase or state of matter to another. Usually, within a phase or state of matter, it has smooth physical properties and the physical variables, such as temperature and concentration, are continuous. Phase transition process plays crucial roles in a variety of scientific, engineering, and industrial processes, including diamond crystal growth[1, the formation of mineral deposits, processing of advanced ceramic materials and coatings[2], weather forecasting[3], melt extraction from Earth's upper mantle [4, 5, 6], geochemical self-organization[7], and environmental contaminant transport [8]. The phase transition problems have been studied by different kinds of approaches: from experimental researches [4, 9, 10, to numerical simulations [11, 12, 13, 14, 3, 15]; from solving partial differential equations [16, 17, 18, 7, 12, 19], to statistical analysis[20, 21, 16, 22], such as random network models to capture the stochastic nature of the phase transition process.

In general, precipitation, deposition, and freezing are unstable processes in which the interface, where phase transition occurs, develops into dendritic fingers, while dissolution, erosion, and melting are just the opposite. The research in this area is aimed at prediction of the detailed evolution of the material interface from the initial shape of the solid particles called seeds, and to estimate macroscopic parameters in coarser space-time scale models. In addition, improved understanding of the physical process in conjunction with experimental measurements are likely to be obtained from modeling and simulating approach that captures the detailed evolution of the solid-liquid interface and enables control of such processes.

For precipitation and dissolution problems, Hartmann et al. studied the dissolution process of solid particles suspended in a turbulent flow by large eddy simulation including passive scalar transport and particle tracking[11]. The successful application of the coupled LES/particle transport/scalar mixing solvers opened worthwhile direction of numerical research like crystallization processes. However, the volume fraction occupied by the particles, which is significant in the first stages of the dispersion, has not been taken into account. Q. Kang et al. developed a lattice-Boltzmann model to simulate dissolution and precipitation in the reactive solid phase in a porous medium, and discussed the effects of Peclet and Peclet-Damköhler numbers on the 
transport and reaction process [14, 13, 23, 24]. The results suggest that lattice Boltzmann model can serve as an reliable quantitative approach to study chemical dissolution in porous media. Dongke Sun et. al. developed a two-dimensional lattice Boltzmann-based model to simulate solutal dendritic growth of binary alloys in the presence of forced flow. The results shows that the solute distribution and dendritic growth are strongly influenced by convection which is consistent with present results. The dendrites grow faster in the upstream direction, but slower in the downstream direction 25. To simulate solidification at microscale, H. Yin et. al. developed an approach which combines the lattice Boltzmann (LB) and the cellular automaton technique (CA) [26]. The results show that the LB-CA algorithm has high computational efficiency when fluid flow is considered. Li Chen et. al. proposed a mesoscopic model based on the lattice Boltzmann method (LBM) to study the precipitation patterns in Liesegang phenomena [27]. In addition, they developed and validated a pore-scale model based on the LBM for multiphase reactive transport with phase transitions and dissolution-precipitation processes. A general LB concentration boundary condition that can handle various concentration boundaries including reactive and moving boundaries with complex geometries is proposed also in [28]. Dijk et. al. investigated the precipitation and dissolution of reactive solutes under the action of fully developed laminar flow in saturated fractures [18]. The results show that the evolution of the solute transport and fracture geometry can be adequately described by the Damköhler and Peclet numbers which can be used as a basis for the development of more complex models of reactive solute transport, precipitation, and dissolution in saturated fractured media. Maisse et al. proposed a mathematical model for dissolution/precipitation with diffusion in a reactive open porous medium and proved the existence and uniqueness of solution, and this model can be used for predictive numerical simulation [29]. Oldham et al. provided computational predictions for local airflow and particle deposition patterns [10]. Koenig described a method of computing the growth rate of ice crystals by deposition, predicted growth characteristics, and compared them with meager experimental data [3]. A simple, empirical equation using two temperature-dependent parameters was found to describe the crystal growth rate as a function of mass. Chang et al. developed a three-dimensional Eulerian regional acid deposition model to calculate episodic chemical concentrations and dry and wet deposition of acids [30]. This model is relatively comprehensive and provides a framework to examine the relative importance and sensitivity of numerous physical and 
chemical processes responsible for the formation and deposition of tropospheric acidity. $\mathrm{Xu}$ and Meakin took a phase-field approach for modeling solute precipitation 31. By using a detailed asymptotic analysis, the connections between the sharp-interface and phase-field models were established which ensures that the phase-field model will converge to the corresponding free-boundary problem.

The freezing and melting problems are widely discussed in weather forecasting and de-icing systems. Huneault et al. described and compared the theory behind three de-icing models: Manitoba Hydro (MH) model, IREQ_1 model and IREQ_2 model. The latter two models expand on a well-known de-icing computation by adding information about ice buildup and ice/water transition on outer and inner surfaces of ice sleeve 32. Lasse Makkonen presented a numerical model which includes detailed simulation of icicle growth, and showed that ice loads formed close $0^{\circ} \mathrm{C}$ may be much higher than those formed at lower temperatures [33]. Epstein et al. presented an overall view of the subject of solid-liquid phase change in fluid flow and showed the possibility to make a reasonably accurate prediction of the pressure drop in turbulent flow [34]. Khvorostyanov et al. studied the liquid-solid phase transitions based on equations which express the properties as functions of temperature, solution molality, pressure and finite size of freezing/melting particles [35]. The results indicated that this approach allows reproduction of measured melting and freezing temperatures as functions of solution molality and pressure over wide ranges for many geophysical applications. Nagai et al. developed a simulation method for calculating heat transfer around a pipe-in-pipe snow melting system and simulated time variation of temperature field and snow depth around the system [15]. By comparing with the experimental data, they concluded that snow melting ability and snow residue situations of this system are predictable. Lozowski et al. described a model which simulated icing on an unheated, non-rotating cylinder, and computed the thermodynamic conditions and the initial icing rate as a function of angle around the upstream face of the cylinder[36]. The model is not constrained to a consideration of stagnation line conditions only, nor is it necessary to limit its application to cases where the accretion is growing dry makes it qualitatively realistic.

In this paper, we focus on the numerical simulations of the interface evolution. The moving interface is an infinitesimally thin hyper-surface that separates adjacent phases. Various interface methods have been proposed and applied to these problems. Among them, the Eulerian level set method 
by Tan and Zabaras [37, 38], the smoothed particle hydrodynamics (SPH) method by Tartakovsky et al [39], and the front tracking method by AlRawahi and Tryggvason[40]. The front tracking method and Lagrangian type SPH method give high resolution to the dendritic structures of solid at large Damköhler number, while the level set method is more suitable for the smooth interface growth, which occurs at low Damköhler number. In our study, the moving boundary between adjacent phases is a thin curve (2D) or surface (3D) of zero thickness. We implement our numerical algorithms for precipitation, dissolution, freezing and melting based on the front tracking data structures and functionality. We study the precipitation process through high resolution tracking both with and without advection flow. We mimic the dissolution of homogeneous and non-homogeneous solid particles immersed in a dilute and incompressible liquid solution. We simulate the freezing and melting problems with realistic physical parameters. The simulations of these phase transition problems show the high resolution and accuracy in handling topological changes by the interface tracking method. It demonstrates the capability of the FronTier library to simulate different types of phase transition problems both in two and three dimensions. The examples shown in this paper demonstrate that our Lagrangian tracking algorithm forms a computational platform capable of studying deposition, dissolution, freezing, and melting both with and without advection due to the surrounding fluid.

\section{Mathematical Model}

\subsection{Mathematical Model for Deposition/Dissolution}

In our first model, the solid phase occupying the domain $\Omega_{p}(t)$ at the time $t[T]$ is immersed in a fluid $\Omega_{f}(t)$. The fluid itself consists of an inert mixture of other species assumed to have no chemical interaction with the solid species, but to interact mechanically as described by a model based on a theory of continuum mixture. The moving boundary between two different phases is an infinitesimally thin curve (2D) or surface (3D). At the boundary, the partial differential equation for the conservation law takes an integral form for weak solution, due to the discontinuity of variables.

The region around the solid contains a solute with spacial concentration $C=C(\mathbf{x}, t)\left[M /\left(L^{3}\right)\right]$, as a function of position and time. If the solute is motionless and the diffusion coefficient $\alpha\left[L^{2} / T\right]$ is a constant, the flux of 
the solute follows Fick's first law $\mathbf{J}=-\alpha \nabla C$ and the solute concentration function satisfies the diffusion equation

$$
\frac{\partial C}{\partial t}=\alpha \Delta C \quad \text { in } \quad \Omega_{f}(t)
$$

When the solute is dissolved in a flowing fluid, the solute will undergo both diffusion and advection. In this case, we add the term $(\mathbf{u} \cdot \nabla) C$ as the advection part. Thus, the solute concentration function satisfies the advection-diffusion equation

$$
\frac{\partial C}{\partial t}+(\mathbf{u} \cdot \nabla) C=\alpha \Delta C \quad \text { in } \quad \Omega_{f}(t)
$$

where $\mathbf{u}[L / T]$ is the fluid velocity (equal to zero in the solid). Here we have assumed that the fluid is incompressible and the viscous heating can be neglected.

Eq. (2) can be solved by finite difference methods, Lattice Boltzmann method or some other methods. The major challenge for computing this problem accurately lies in the propagation of the phase boundary and the coupling between the phase boundary and the PDE solver for the interior of subdomains. The propagation of the solid boundary is determined by the conservation of mass, that is, the mass transfer from liquid phase (solute) to solid phase (crystal) in deposition and vice versa in dissolution.

In our simulation, only the momentum balance associated to the motion of the fluid mixture as a whole is considered. This is in contrast to a more rigorous approach wherein the momentum balance for each species is taken into account. This simplification avoids theoretical considerations of stress-deformation relations that heretofore lack experimental validation. The velocity field of the solute is solved from the Navier-Stokes equation:

$$
\frac{\partial \mathbf{u}}{\partial t}+(\mathbf{u} \cdot \nabla) \mathbf{u}=-\frac{1}{\rho} \nabla p+\frac{\mu}{\rho} \nabla^{2} \mathbf{u} \quad \text { in } \quad \Omega_{f}(t),
$$

where $\mu[M /(L T)]$ is the viscosity, $p\left[M /\left(L T^{2}\right)\right]$ is the pressure, and $\rho\left[M / L^{3}\right]$ is the fluid density. The incompressibility is described by the divergence-free condition

$$
\nabla \cdot \mathbf{u}=0 \quad \text { in } \quad \Omega_{f}(t)
$$

Denote the subdomain occupied by solid $\Omega_{p}(t)$, and the fluid field as $\Omega_{f}(t)$. Let the mass transfer between $\Omega_{p}(t)$ and $\Omega_{f}(t)$, through the interface represented by the surface $\partial \Omega_{p}(t)$. Denoting by $C_{e}$ the equilibrium concentration 
of solute at the interface, we assume that the rate of mass condensation from solute in liquid to the solid crystal can be locally measured by the reaction rate $k[L / T]$, and therefore the condensed crystal mass can be obtained from $k\left(C\left(\mathbf{x}_{s}\right)-C_{e}\right)$ at every point $\mathbf{x}_{s} \in \partial \Omega_{p}(t)$ on surface, where $C\left(\mathbf{x}_{s}\right)$ is the local concentration of the solute at the solid interface. Let $\rho_{s}\left[M / L^{3}\right]$ denote the mass density of the solid (crystal), according to the local mass conservation, the following equation describes the interfacial mass balance in a small time increment $d t[T]$.

$$
\rho_{s} d S d h=k\left(C\left(\mathbf{x}_{s}\right)-C_{e}\right) d S d t
$$

where $d h[L]$ is an infinitesimal displacement of the surface $\partial \Omega_{p}(t)$ measured in the direction of the outward pointing vector from the solid into the liquid. The element of surface area is represented by $d S\left[L^{2}\right]$, and $d t[T]$ is the time differential. Figure 1 shows the mathematical surface representation of the interface.

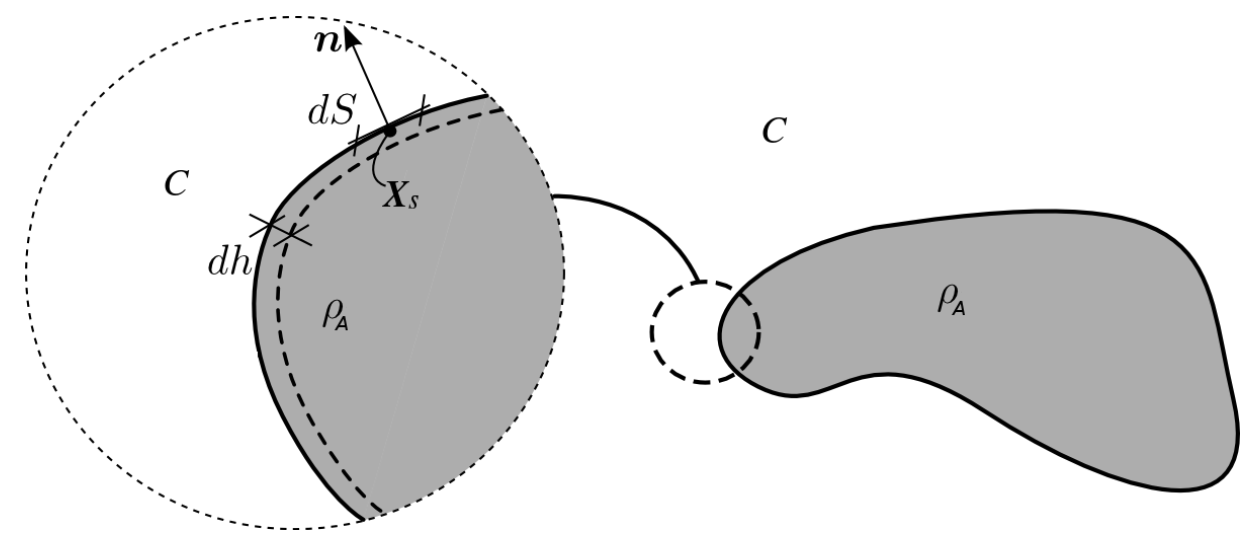

Figure 1: Mathematical surface representation of the interface.

The normal speed of the interface can be obtained from Eq. (5) as

$$
v_{n}:=\frac{d h}{d t}=\frac{k\left(C\left(\mathbf{x}_{s}\right)-C_{e}\right)}{\rho_{s}} .
$$

If we use the first-order kinetic reaction model at the solid-fluid interface, we will have

$$
\alpha \frac{d C}{d n}\left(\mathbf{x}_{s}\right)=k\left(C\left(\mathbf{x}_{s}\right)-C_{e}\right)
$$


where $\frac{d C}{d n}$ is derivative of $C$ along the direction normal to the interface toward the fluid phase. The propagation speed of the interface can then be obtained by

$$
v_{n}=\frac{\alpha}{\rho_{s}} \frac{d C}{d n}\left(\mathbf{x}_{s}\right) .
$$

In a saturated solute, that is $C\left(\mathbf{x}_{s}\right)>C_{e}$, the solute tends to condense on the solid interface. While in an unsaturated solute, that is $C\left(\mathbf{x}_{s}\right)<C_{e}$, the solid substance tends to dissolve into the solvent. Since some of the chemical reactions are irreversible, the interface solver may allow dual-directional propagation (deposition and dissolution) or uni-directional propagation (deposition or dissolution only).

\subsection{Mathematical Model for Freezing/Melting}

We use Kelvin as the unit for measurement of temperature in our numerical simulations, and denote the temperature by $T[\Theta]$. Given the temperature gradient $\nabla T[\Theta / L]$, by Fourier's law, the heat flux is a vector whose direction is opposite to the temperature gradient, that is $\mathbf{q}=-\kappa \nabla T$, where $\kappa\left[M L /\left(\Theta T^{3}\right)\right]$ is the thermal conductivity. By conservation of thermal energy (heat), we have

$$
C_{p} \rho \frac{\partial T}{\partial t}+\nabla \cdot \mathbf{q}=0
$$

That is,

$$
C_{p} \rho \frac{\partial T}{\partial t}-\nabla \cdot(\kappa \nabla T)=0,
$$

where $C_{p}\left[L^{2} /\left(T^{2} \Theta\right)\right]$ is the specific heat with constant pressure, and $\rho\left[M / L^{3}\right]$ is the mass density of the material. Denote the temperature of solid phase and liquid phase by $T_{s}$ and $T_{l}$ respectively in two subdomain separated by the phase transition interface, we have

$$
\frac{\partial T_{i}}{\partial t}=\frac{\kappa_{i}}{C_{p}^{i} \rho_{i}} \Delta T_{i}=\alpha_{i} \Delta T_{i}, \quad i=s, l
$$

where $\alpha_{i}=\frac{\kappa_{i}}{C_{p}^{i} \rho_{i}}$ is the diffusion coefficient in Eq. (11).

If the water liquid phase is flowing, the freezing/melting process will have an advection term in the equation. In the latter case, the temperature in the 
liquid phase satisfies

$$
\frac{\partial T_{l}}{\partial t}+(\mathbf{u} \cdot \nabla) T_{l}=\frac{\kappa_{l}}{C_{p}^{l} \rho_{l}} \Delta T_{l}=\alpha_{l} \Delta T_{l} .
$$

The temperature in the solid phase always satisfies the diffusion-only equation

$$
\frac{\partial T_{s}}{\partial t}=\frac{\kappa_{s}}{C_{p}^{s} \rho_{s}} \Delta T_{s}=\alpha_{s} \Delta T_{s} .
$$

At the phase interface, let $\frac{\partial T_{s}}{\partial n}$ and $\frac{\partial T_{l}}{\partial n}$ denote the normal gradient of temperature on the solid side and liquid side respectively. The rate of net heat deposited at the interface is

$$
H=\kappa_{s} \frac{\partial T_{s}}{\partial n}-\kappa_{l} \frac{\partial T_{l}}{\partial n},
$$

This net heat should equal to the latent heat to either freeze or melt (depending on the sign of $H$ ) the material at the interface. Let $\rho_{s}$ be the mass density of solid material, the energy balance at the ice-water interface can be written as

$$
\rho_{s} L v_{n}=H,
$$

where $L$ is the latent heat, $v_{n}$ is the normal propagation speed of the interface. Therefore, the interface propagation speed $v_{n}$ can be written as follows,

$$
v_{n}=\frac{H}{\rho_{s} L} .
$$

\section{Dimensionless Control Parameters}

Dimensional analysis suggests that dimensionless parameters, such as Damköhler number $D a$ and Peclet number $P e$, control the phase transition processes [13, 12, 16, 18. The evolution follows similarity law. The solutions are invariant when these dimensionless parameters are equal. In this section we present the dimensional analysis for precipitation, dissolution, freezing and melting.

Let $T_{c}[T]$ be the characteristic time and $L_{c}[L]$ be the characteristic length, then the characteristic velocity $\mathbf{U}_{c}[L / T]$ satisfies $\mathbf{U}_{c}=\frac{L_{c}}{T_{c}}$. And let $\tilde{t}=\frac{t}{T_{c}}$, 
$\tilde{\mathbf{x}}=\frac{\mathbf{x}}{L_{c}}$ be the dimensionless time and spatial coordinates. For precipitation/dissolution problems, the solute concentration equations can be reduced to

$$
\begin{aligned}
\frac{\partial C}{\partial \tilde{t}} & =\frac{\alpha T}{L^{2}} \tilde{\Delta} C, \\
\frac{\partial C}{\partial \tilde{t}}+(\tilde{\mathbf{u}} \cdot \tilde{\nabla}) C & =\frac{\alpha T}{L^{2}} \tilde{\Delta} C .
\end{aligned}
$$

where $\tilde{\mathbf{u}}=\frac{\mathbf{u}}{\mathbf{U}_{c}}=\frac{T_{c}}{L_{c}} \mathbf{u}$, is the velocity vector in dimensionless coordinates, $\tilde{\nabla}$ and $\tilde{\Delta}$ are the differential operators in dimensionless coordinates. Eq. (17) is independent of time and length units if we consider the dimensionless diffusion coefficient as

$$
\tilde{\alpha}=\frac{\alpha T_{c}}{L_{c}^{2}} .
$$

In solute precipitation, the Damköhler number is defined as $D a:=\frac{k L_{c}}{\alpha}$, and the Peclet number is defined as $P e:=\frac{\mathbf{U}_{c} L_{c}}{\alpha}$ [41. The Damköhler number describes the effect of deposition rate relative to the solute diffusion rate. The Peclet number describes the effect of convection rate relative to the diffusion rate on the solute transport.

For freezing/melting problems, the equations describing the temperature field can also be transformed into

$$
\begin{aligned}
\frac{\partial T}{\partial \tilde{t}} & =\frac{\alpha T_{c}}{L_{c}^{2}} \tilde{\Delta} T, \\
\frac{\partial T}{\partial \tilde{t}}+(\tilde{\mathbf{u}} \cdot \tilde{\nabla}) T & =\frac{\alpha T_{c}}{L_{c}^{2}} \tilde{\Delta} T .
\end{aligned}
$$

where the thermal diffusivity $\alpha$ is defined as $\alpha=\frac{\kappa}{C_{p} \rho}, \kappa$ is the thermal conductivity, $C_{p}$ is the specific heat, $\rho$ is the material mass density, $\tilde{\mathbf{u}}=\frac{T_{c}}{L_{c}} \mathbf{u}$ is the velocity vector in dimensionless coordinates, $\tilde{\nabla}$ and $\tilde{\Delta}$ are the differential operators in dimensionless coordinates. Eq. (19) is independent of time and length units if we consider the dimensionless thermal diffusion coefficient as

$$
\tilde{\alpha}=\frac{\alpha T_{c}}{L_{c}^{2}}=\frac{\kappa}{C_{p} \rho} \frac{T_{c}}{L_{c}^{2}} .
$$

In freezing problems, the Peclet number for heat is also defined as $P e:=$ $\frac{\mathbf{U}_{c} L_{c}}{\alpha}$, where the thermal diffusivity $\alpha$ is $\frac{\kappa}{C_{p} \rho}$. The Peclet number for heat 
reveals the rate of advection of temperature by the fluid flow relative to the diffusion of temperature.

\section{Numerical Methods}

The computational procedure for simulating phase transition problems is demonstrated by the flow-chart in Figure 2. The numerical system we have established for the simulations includes several components. The geometrical data structures and functionality are from the FronTier library [42], which was developed based on the front tracking method for the study of fluid instabilities. In order to add the advection term $(\mathbf{u} \cdot \nabla)$ when there is a fluid flow, we compute the velocity from the incompressible Navier-Stokes solver.

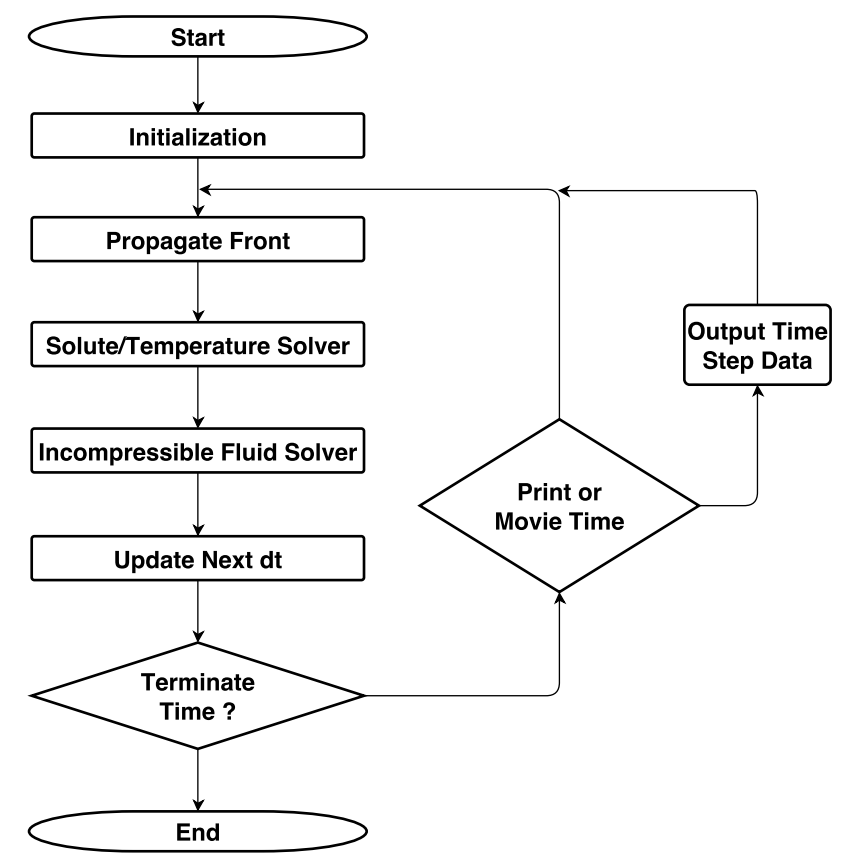

Figure 2: Flow chart for the phase transition simulations in the FronTier code.

\subsection{Lagrangian Front Tracking}

The Lagrangian front tracking method is an adaptive computational method to follow the dynamic evolution of a moving interface across which physical variables are discontinuous. It propagates the interface position as a 

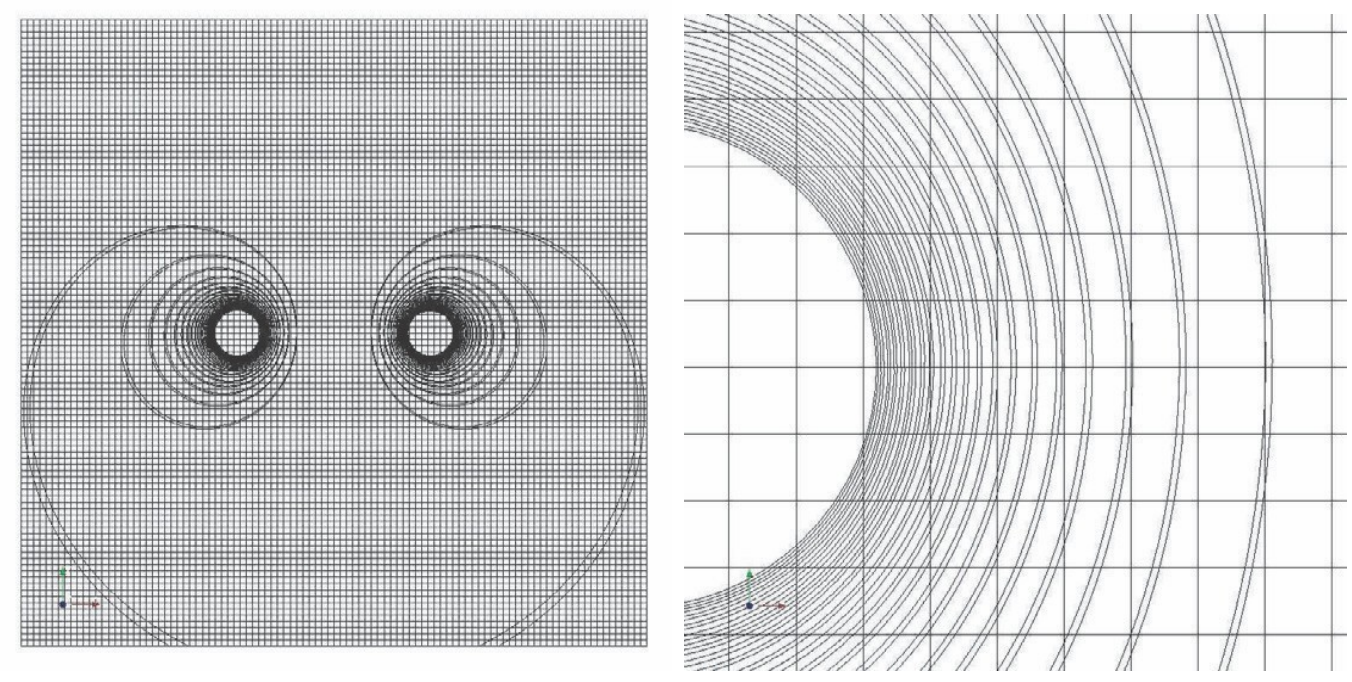

Figure 3: 2D Benchmark test on subgrid resolution in FronTier. The right plot is a blowup which shows that in theory, the resolution of front can be as high as 1/1000 of the grid spacing. But when coupled with the PDE solver, the accuracy of resolution is limited by the truncation errors of the numerical scheme.

set of topologically connected marker points and then applies finite difference method to each subdomain where the physical variables are relatively smooth. The interface is treated as an interior boundary for the PDE solver in each subdomain. This method gives high resolution at subgrid level and maintains sharp corners with acute angles [43, 44]. Figure 3 shows that FronTier library can resolve the interface as high as 1/1000 of grid spacing.

The FronTier library can also handle geometrical and topological changes of the front robustly. Figure 4 shows the capability of the FronTier library in handling 3D geometrical and topological changes of the surface mesh such as twisting, merging and bifurcation of the three dimensional surfaces.

When the front velocity depends on the geometry of the interface, an accurate computation of interface geometric variables is needed. The second order normal or curvature calculation in FronTier is based on the numerical framework developed by Jiao et al [45, 46]. In this algorithm, a local coordinate system is constructed with a height function, and local polynomial fitting is performed based on Taylor expansion to obtain gradient and Hessian of the height functions. The solute/temperature gradient $\partial C / \partial n$ or $\partial T / \partial n$ at each interface point is calculated under this framework by performing the 


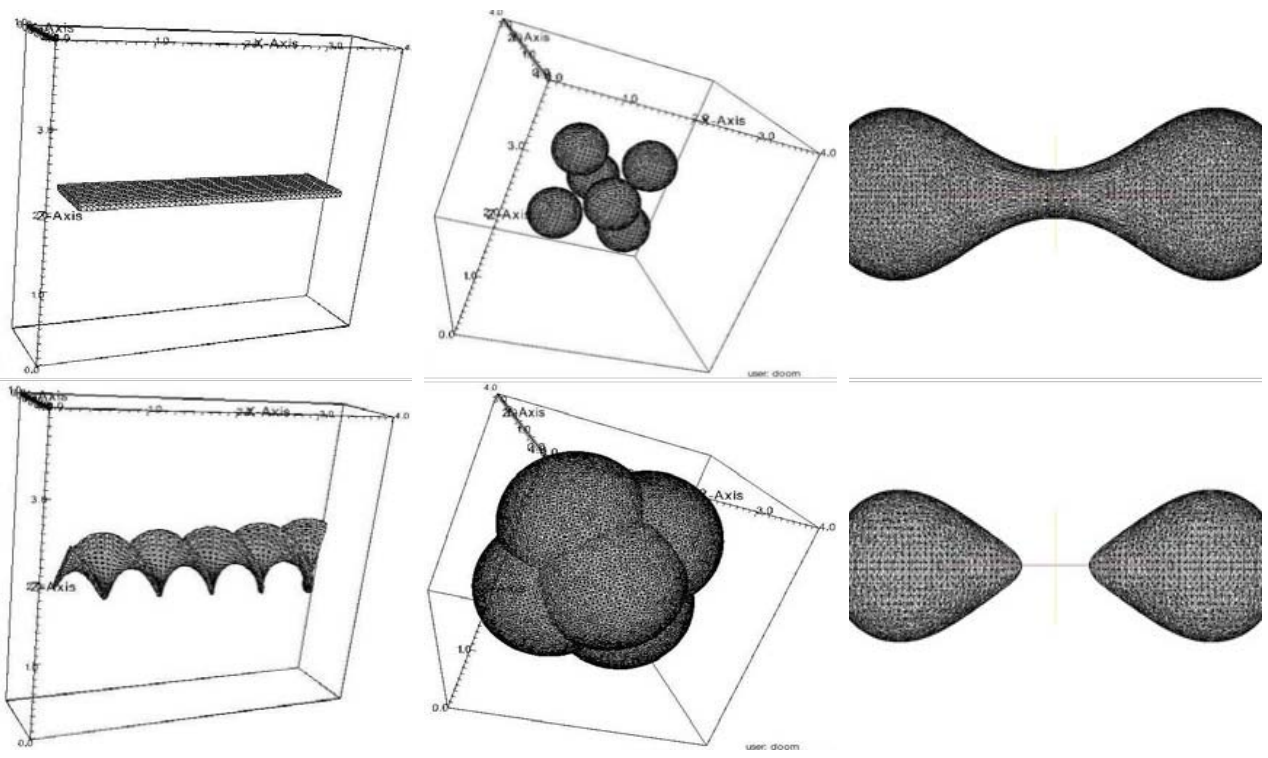

Figure 4: Examples of 3D FronTier benchmark simulations. From left to right: twisting, merging, and bifurcation of the three dimensional surface mesh.

finite difference of the interpolated variables (solute or temperature) along the normal direction of the interface point. The left plot of Figure 5 shows such calculation.

\subsection{Finite Difference Methods for Solute/Temperature Domain}

We choose FronTier functionality to simulate phase transition process for two reasons. First, the velocity of the moving interface is localized to the interface itself. An Eulerian method, such as the level set method, requires extrapolation of the velocity to advance the level function in space and interpolation to determine the interface position. The extrapolation and interpolation not only add to the computational cost, but also introduce diffusive effect to artificially smooth the interface. Second, the Lagrangian method is not constrained by the supporting computational mesh. It can achieve higher subgrid resolution. Since front tracking has an explicit representation of the interface, the computation of surface related physical processes, such as deposition, diffusion, and chemical reactions are natural in this framework [47].

For the solute/temperature domain, central difference schemes are used for the parabolic diffusion equation, these include the explicit scheme, the 


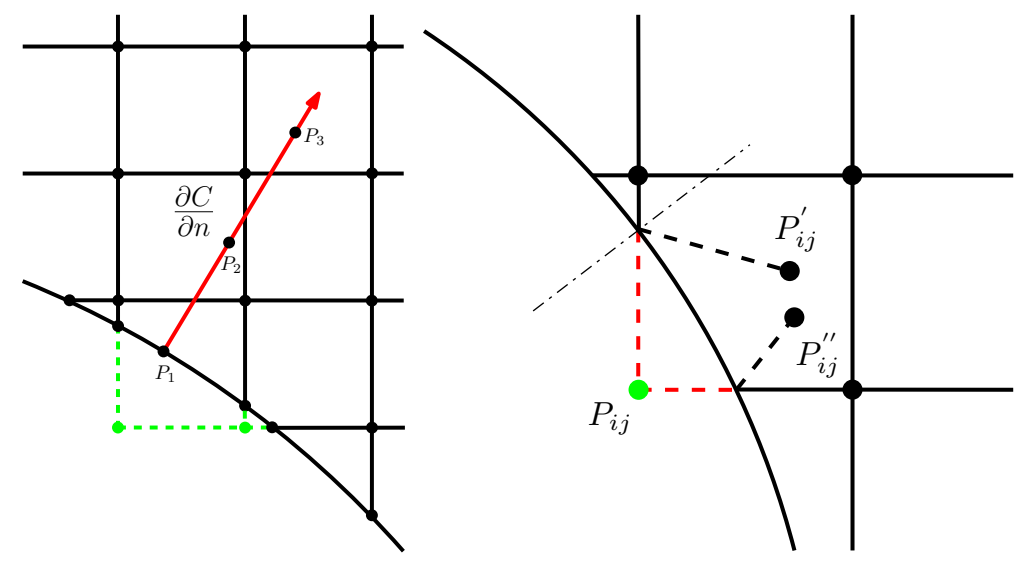

Figure 5: Numerical discretization at the irregular boundary. The left plot shows the algorithm for point propagation and the right plot shows the reflection and interpolation algorithm for the interior solver. The calculation of normal direction and reflection both have second order accuracy in interface propagation and finite difference solver. The FronTier ++ library supports the interpolation functions near the boundary for a second order calculation of normal derivative as well as finite difference solver.

implicit scheme and the Crank-Nicolson scheme. The explicit scheme is first order in $\Delta t$ and second order in $\Delta x$. However, because of the CFL condition for parabolic PDE, $\Delta t \sim \Delta x^{2}$, the scheme is effectively second order in both $\Delta t$ and $\Delta x$. The implicit scheme uses 2 nd order Runge-Kutta scheme in time to achieve second order in $\Delta t$ and second order in $\Delta x$. The CrankNicolson scheme is second order on both $\Delta x$ and $\Delta t$. Each scheme has its pros and cons. The implicit scheme and Crank-Nicolson scheme are both unconditionally stable. They result in a linear system which needs be solved by public domain software package such as PETSc. The implicit schemes can take relatively larger time step, which is more efficient under mesh refinement, especially in three dimensional simulations, while the explicit scheme is slow due to stiff CFL condition $\Delta t \sim \Delta x^{2}$. But its small time step allows more stable front propagation and more steps for interface mesh optimization [47. Most of the 2D simulations with no fluid flow are carried out by using the central explicit scheme.

With fluid flow of the liquid phase, we must consider the advection term in Eq. (2) and Eq. (12). For the explicit scheme, since central difference scheme for advection term is unconditionally unstable, therefore we use upwind scheme for the advection term. In $d$ dimensions, let us denote $\mathbf{u}=\left(u_{1}, u_{2}, \cdots\right)$ as the velocity of the flowing fluid, $C$ as the solute concentra- 
tion in mass transfer or the temperature in heat transfer, the discretization for the advection-diffusion equation is

$$
C^{n+1}=C^{n}-\sum_{k=1}^{d} \frac{\Delta t}{\Delta x_{k}}\left(u_{k}^{+} \delta_{k}^{-} C^{n}+u_{k}^{-} \delta_{k}^{+} C^{n}\right)+\alpha \sum_{k=1}^{d} \frac{\Delta t}{\Delta x_{k}^{2}} \delta_{k}^{2} C^{n}
$$

where $u_{k}^{+}=\max \left(0, u_{k}\right), u_{k}^{-}=\min \left(0, u_{k}\right)$ for $k=1, \cdots, d$. And $\delta_{k}^{-}, \delta_{k}^{+}$, and $\delta_{k}^{2}$ are the backward, forward and second order central difference operators respectively. For the implicit scheme, we use second order Runge-Kutta method to discretize the time step. The formula is

$$
\begin{aligned}
C^{*} & =C^{n}+\Delta t f\left(C^{*}\right), \\
C^{n+1} & =C^{n}+\frac{1}{2} \Delta t f\left(C^{*}\right)+\frac{1}{2} \Delta t f\left(C^{n+1}\right),
\end{aligned}
$$

where

$$
f(C)=-\sum_{k=1}^{d} \frac{\Delta t}{2 \Delta x_{k}} u_{k} \delta_{k}^{0} C+\alpha \sum_{k=1}^{d} \frac{\Delta t}{\Delta x_{k}^{2}} \delta_{k}^{2} C .
$$

Here, $\delta_{k}^{0}$ is the first order central difference operator. For the Crank-Nicolson scheme, the discretization for the advection-diffusion equation is

$$
C^{n+1}=C^{n}-\frac{1}{2} \sum_{k=1}^{d} \frac{\Delta t}{2 \Delta x_{k}} u_{k}\left(\delta_{k}^{0} C^{n+1}+\delta_{k}^{0} C^{n}\right)+\frac{1}{2} \alpha \sum_{k=1}^{d} \frac{\Delta t}{\Delta x_{k}^{2}}\left(\delta_{k}^{2} C^{n+1}+\delta_{k}^{2} C^{n}\right),
$$

where $\delta_{k}^{0}$ and $\delta_{k}^{2}$ are the first order and second order central difference operators respectively. At the irregular boundary of the domain such as the crystal-solute interface, we use the point reflection and interpolation in the cell at which the reflected point is landed. This method provide second order accuracy for the Crank-Nicolson scheme at the boundary. This algorithm is applied to both the solute solver and the fluid solver. The right plot of Figure 5 illustrates such numerical treatment.

\subsection{Projection Method for Incompressible Navier-Stokes}

For single phase incompressible fluid, the vector form of Navier-Stokes equations are given by:

$$
\mathbf{u}_{t}+(\mathbf{u} \cdot \nabla) \mathbf{u}=-\frac{1}{\rho} \nabla p+\frac{\mu}{\rho} \nabla^{2} \mathbf{u}+\mathbf{f}+\mathbf{g},
$$




$$
\nabla \cdot \mathbf{u}=0
$$

We implemented the incompressible fluid solver in the FronTier application module based on the projection method[48]. It consists of four main steps, which are shown below:

1) Compute Advection equation. The calculation of advection of the velocity field is by solving the equation:

$$
\mathbf{u}_{t}+(\mathbf{u} \cdot \nabla) \mathbf{u}=0
$$

The spacial discretization is based on the fifth-order finite difference WENO scheme by Jiang and Shu[49] with a framework for the design of smoothness indicators and nonlinear weights. The WENO scheme uses the idea of adaptive stencils to automatically achieve high order accuracy and non-oscillatory property near discontinuities. The time discretization is implemented by the fourth order TVD Runge-Kutta methods:

$$
\begin{aligned}
\mathbf{u}^{(1)} & =\mathbf{u}^{n}+\frac{\Delta t}{2} \mathcal{L}\left(\mathbf{u}^{n}\right) \\
\mathbf{u}^{(2)} & =\mathbf{u}^{n}+\frac{\Delta t}{2} \mathcal{L}\left(\mathbf{u}^{(1)}\right) \\
\mathbf{u}^{(3)} & =\mathbf{u}^{n}+\Delta t \mathcal{L}\left(\mathbf{u}^{(2)}\right) \\
\mathbf{u}^{*} & =-\frac{1}{3} \mathbf{u}^{n}+\frac{1}{3} \mathbf{u}^{(1)}+\frac{2}{3} \mathbf{u}^{(2)}+\frac{1}{3} \mathbf{u}^{(3)}+\frac{\Delta t}{6} \mathcal{L}\left(\mathbf{u}^{(3)}\right)
\end{aligned}
$$

The advection equation is a scalar equation with both linear and nonlinear flux functions. The WENO scheme gives high resolution to the velocity field, especially at large Reynold number.

2) Compute diffusion equation. To solve the following equation,

$$
\frac{\mathbf{u}^{* *}-\mathbf{u}^{*}}{\Delta t}=\frac{\mu}{\rho} \nabla^{2} \mathbf{u}+\mathbf{f}+\mathbf{g}-\frac{1}{\rho} \nabla p .
$$

We use second order Crank-Nicolson scheme for the diffusion term, and treat the $\mathbf{f}+\mathbf{g}-\frac{1}{\rho} \nabla p$ as the source terms.

3) Projection Method. We compute the projection by solving the Poisson's equation

$$
\Delta \phi=\nabla \mathbf{u}^{* *}
$$


Then, the pressure can be updated by

$$
p^{n+\frac{1}{2}}=p^{n-\frac{1}{2}}+\phi .
$$

4) Update velocity. Finally, we update the velocity in new time step by

$$
\mathbf{u}^{n+1}=\mathbf{u}^{* *}-\nabla \phi .
$$

Then $\mathbf{u}^{n+1}$ satisfies the divergence-free requirement.

The verification and validation of above algorithms has been presented in [43, 47] et al.

\section{Numerical Results for Precipitation}

For solute precipitation, we focus on the study of crystal growth. Crystal growth is an unstable process. It comes with the surface roughening due to amplification of small perturbations generated by numerical anisotropy in the precipitation process. The gradient of solute concentration at the convex part of the interface is larger than the gradient of solute concentration at the concave part of the interface. Consequently, the crystal growth follows "winner takes it all" principle, that is, the more convex is the front, the higher solute gradient it encounters, thus the faster it grows. The concave part of the front stays in the region where the solute is depleted and eventually stops growing. It is such unstable front competition that results in the dendritic structure of the interface. In our simulations, the unstable growth is initialized by a small perturbation to the front. However, even a truncation error can start the unstable growth from a smooth front.

\subsection{Experiment platform}

All the studies in this work were implemented on a dell precision T7600 Workstation with dual Intel Xeon E5-2687W CPUs. The Intel Xeon E5$2687 \mathrm{~W}$ CPU is the latest multi-threaded multi-core Intel-Architecture processor. It offers eight cores on the same die running at $3.10 \mathrm{GHz}$. The Intel Xeon E5-2687W processor cores feature an out-of-order super-scalar microarchitecture, with newly added 2-way hyper-threading. In addition to scalar units, it also has 4 -wide SIMD units that support a wide range of SIMD instructions. Each has a separate $32 \mathrm{~KB}$ L1 cache for both instructions and data and a $256 \mathrm{~KB}$ unified L2 data cache. All eight cores share an $20 \mathrm{MB}$ L3 data cache. The Intel Xeon E5-2687W processor also features an on-die memory controller that connects to four channels of DDR memory. Fedora 18 with kernel 3.9.2-200 and GCC 4.7.2 were used in the computations. 

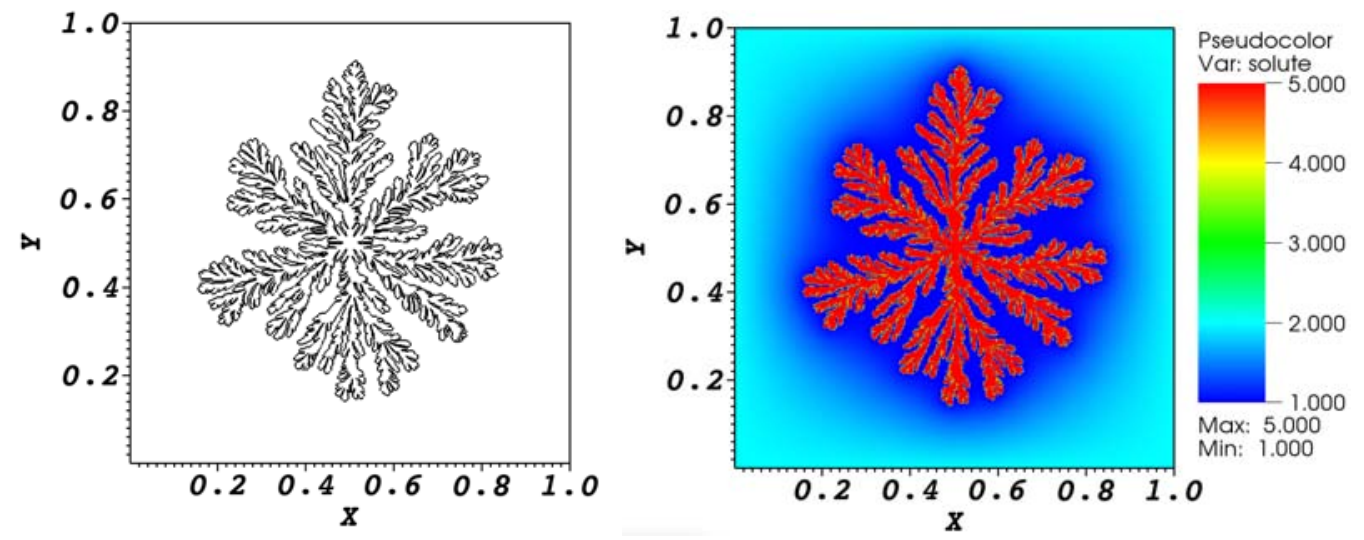

Figure 6: $2 \mathrm{D}$ precipitation from space seed at time $t=1.50$. The initial ambient concentration is $C(t=0)=2.0$. The equilibrium solubility is $C_{e}=1.0$. The mass density of the solid is $\rho_{s}=5.0$. The diffusion coefficient is $\alpha=0.033$ and the reaction rate is $k=50$. The Damköhler number is $D a=1500$. The computational grid is $720 \times 720$. The fractal structure continues to emerge as the computational grid is refined. But the the fractal dimension of the crystal becomes almost constant (in this case $\operatorname{Dim} \approx 1.7$ ) when the grid refinement reaches $160 \times 160$.

\subsection{Precipitation in Saturated Solution}

We conducted two-dimensional simulation of a single seed precipitation in a square domain, $(x, y) \in[0,1] \times[0,1]$. We use Dirichlet boundary conditions on both sides in $\mathrm{x}$ and $\mathrm{y}$ directions. The initial condition in the ambient area is set to be $C(t=0)=2.0$, and the equilibrium solubility $C_{e}$ is set to be 1.0. The precipitation seed is a perturbed circle located in the center of the square domain, whose density is set as $\rho_{s}=5.0$. The radius of this perturbed circle is given by $r=0.01-0.003 \sin \left(6 \theta+\frac{1}{18} \pi\right)$, where $0<\theta<2 \pi$. Here the diffusion coefficient and the reaction rate are set to $\alpha=0.033$ and $k=50$, respectively. In this case, the Damköhler number is $D a=1500$. The numerical scheme used for this simulation is explicit to accurately track the dendritic growth. Figure 6 shows the precipitation interface(left) and the solute concentration in the liquid(right) at time $t=1.50$.

The numerical simulation of deposition process shows the high resolution of dendritic structure in crystal growth. As the crystal interface propagates, the solute concentration in the concave valley of the interface is quickly depleted and reaches equilibrium with the concentration at the crystal surface. Consequently, the crystal growth in this part is stopped. While at the convex front of the interface, the high gradient of solute concentration makes the 
crystal grow faster. The simulation is performed simultaneously on 9 CPUs, and it takes 1 hour 7 minutes and 40 seconds.

Two 3D simulations are performed herein. In the first case, we simulate the precipitation with Dirichlet boundary in all directions. The solute concentration at each boundary is 2.0. The initial precipitation seed is a perturbed sphere, whose radius is given by $r=0.03+0.005 * \sin (4 \theta+1.5 \pi) *$ $\cos (\phi)$, where $0<\theta<2 \pi$, and $0<\phi<\pi$, located at the center of the 3D computational domain $(x, y, z) \in[0,1] \times[0,1] \times[0,1]$. The diffusion coefficient and the reaction rate are set to $\alpha=0.5$ and $k=5000$. In this case, the Damköhler number is $D a=10000$. The initial condition in the ambient area is set to be $C(t=0)=2.0$, and the equilibrium solubility $C_{e}$ is set to be 1.0. The crystal density is $\rho_{s}=5$. In the second case, we simulate the precipitation with Dirichlet boundary in $x$, and $y$ directions, and Neumann boundary in $z$ direction. The same seed located at the center of the 3D computational domain $(x, y, z) \in[0,1] \times[0,1] \times[0.46875,0.53125]$. All other parameters are same with the first case. The solute concentration at Dirichlet boundaries is 2.0. We use implicit scheme in this $3 \mathrm{D}$ simulation in order to simulate more efficiently. Figure 7 shows 3D propagation of the solid interface of the first simulation at time $t=0.02,0.06,0.1$. Figure 8 shows the results of the second simulation at time $t=0.0013,0.02,0.04$, respectively. The first case is implemented parallely on $4 \mathrm{CPUs}$, and it takes 11 hours 40 minutes and 39 seconds. The second case is also implemented parallely on 4 CPUs, and it takes about 11 hours and 48 minutes.

\subsection{Damköhler Number Effect}

To study the dependence of the solute precipitation process on Damköhler

number $D a=\frac{k L_{c}}{\alpha}$, we conducted a series of two-dimensional simulations based on different values of diffusion coefficient $\alpha$ and reaction rate $k$.

We first studied the effect of reaction rate on the precipitation of an initially perturbed circle located at the center of a square domain $(x, y) \in$ $[0,1] \times[0,1]$ with radius defined by $r=0.01-0.003 \sin \left(6 \theta+\frac{1}{18} \pi\right)$, where $0<$ $\theta<2 \pi$. The density of the solid is $\rho_{s}=5.0$. And we set boundary conditions in all directions as Dirichlet boundary with constant solute concentration as 2.0, which is the same as the ambient concentration. The equilibrium solubility in the solution is $C_{e}=1.0$. And the initial solute concentration in the ambient area is $C(t=0)=2.0$. We fix the diffusion coefficient $\alpha=0.1$ while increasing the reaction rate. Figure 9 shows the sequence of three simulations with reaction rate $k=50,100,150$ respectively. And we use the 

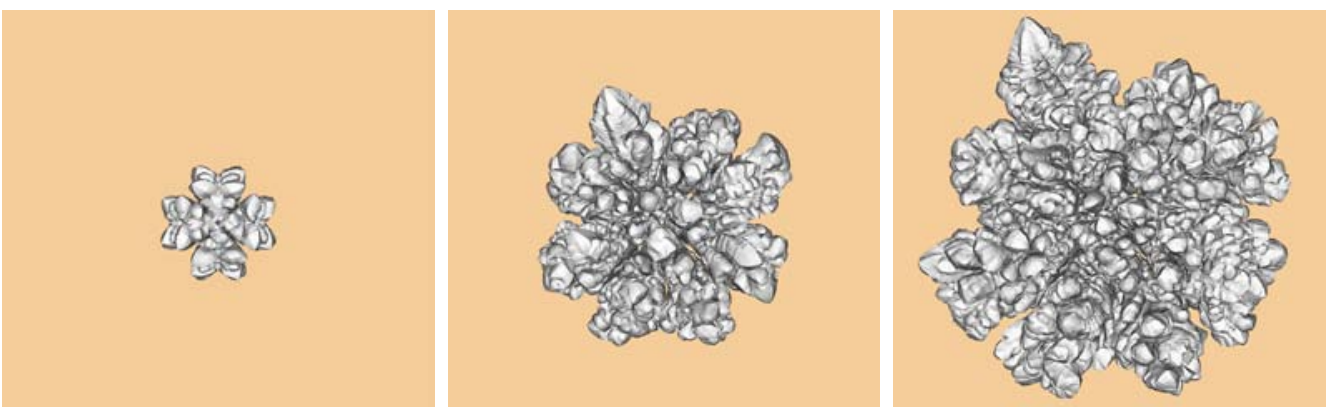

Figure 7: Simulation of 3D precipitation with solid density $\rho_{s}=5$. The initial ambient concentration is $C(t=0)=2.0$. The equilibrium solubility is $C_{e}=1.0$. The diffusion coefficient is $\alpha=0.5$ and the reaction rate is $k=5000$. The Damköhler number is $D a=$ 10000. From left to right, the plots shows the results of simulations with Dirichlet boundary in all directions at time $t=0.02,0.06,0.1$. The computational grid is $128 \times 128 \times 128$. In this case, the solid interface is not as dendritic as appears in Figure 6 due to the coarse computational grid comparing to the $2 \mathrm{D}$ case.
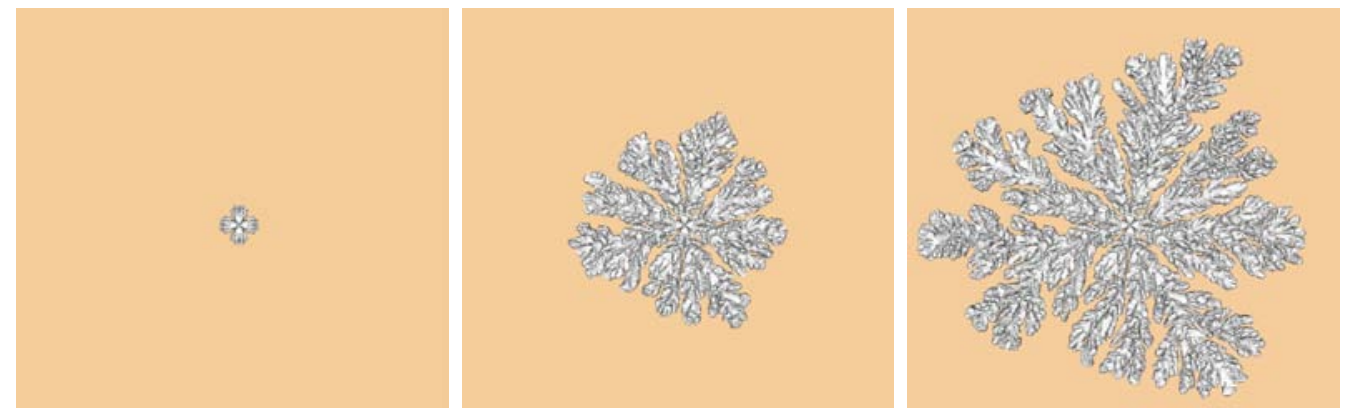

Figure 8: Simulation of psudo-3D precipitation with solid density $\rho_{s}=5$. The initial ambient concentration is $C(t=0)=2.0$. The equilibrium solubility is $C_{e}=1.0$. The diffusion coefficient is $\alpha=0.5$ and the reaction rate is $k=5000$. The Damköhler number is $D a=10000$. From left to right, the plots shows the results of simulations with Dirichlet boundary in $x$ and $y$ directions, and Neumann boundary in $z$ direction at time $t=0.0013,0.02,0.04$. The simulation is in a semi-3D domain of $(0,1) \times(0,1) \times(0,0.0703)$ with Dirichlet boundary in $x$ and $y$ directions, and Neumann boundary in $z$ direction. The computational grid is $512 \times 512 \times 32$. 

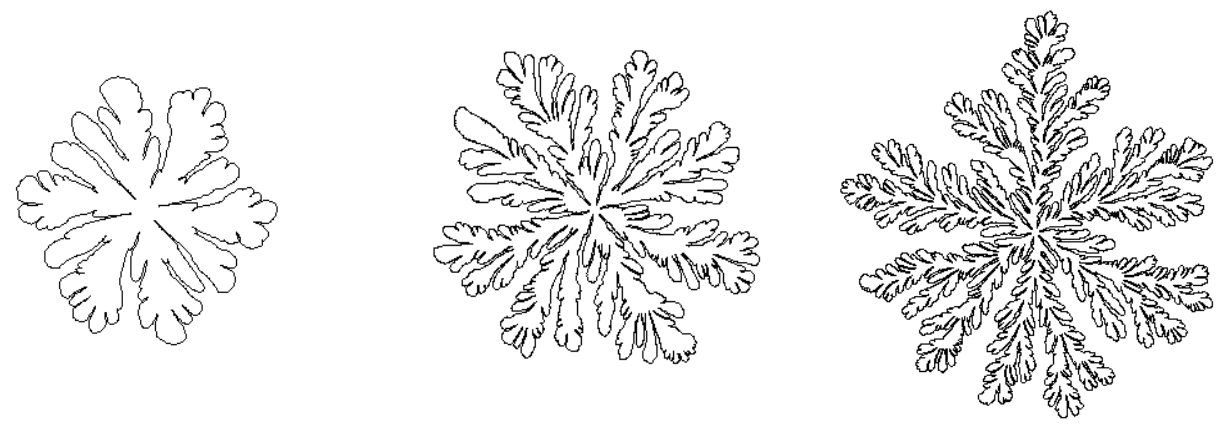

Figure 9: The dendritic structure of precipitation with varying reaction rate $k$ at time $t=0.4$. From left to right: $k=50,100,150$. $D a=500,1000,1500$. The computational grid is $720 \times 720$.

box-counting method to measure the fractal dimension of these three cases which gives $D_{f}=1.895,1.854$, and 1.847 respectively at time $t=0.4$.

In case of larger reaction rate $k$, from Eq. (6), the interface propagation speed is faster, and thus the solid grows more quickly than the case with lower reaction rate. Due to the larger reaction rate $k$, the solute concentration is close to equilibrium concentration at the interface. Therefore, the local gradient of solute concentration is higher near the interface, which results in more dendritic structure.

We also performed a series of simulations to study the effect of diffusion coefficient on precipitation process with the same initial and boundary conditions as those simulations in Figure 9. Here, we fix the reaction rate $k=50$ while decreasing the diffusion coefficient. Figure 10 shows the sequence of three simulations with diffusion coefficient $\alpha=0.1,0.05,0.033$ respectively. And we use the box-counting method to measure the fractal dimension of these three cases which gives $D_{f}=1.895,1.885$, and 1.878 respectively at time $t=0.4$.

In case of smaller diffusion coefficient $\alpha$, from Eq. (8), the interface propagation speed is smaller, and thus the solid grows more slowly than the case with higher diffusion coefficient. Due to the lower diffusion coefficient, the solute concentration at the interface is also very close to equilibrium concentration, and then the local gradient of solute concentration near the interface is larger than the average gradient, which results in more unstable and highly dendritic interface.

The numerical experiments for both increased reaction rate and decreased 

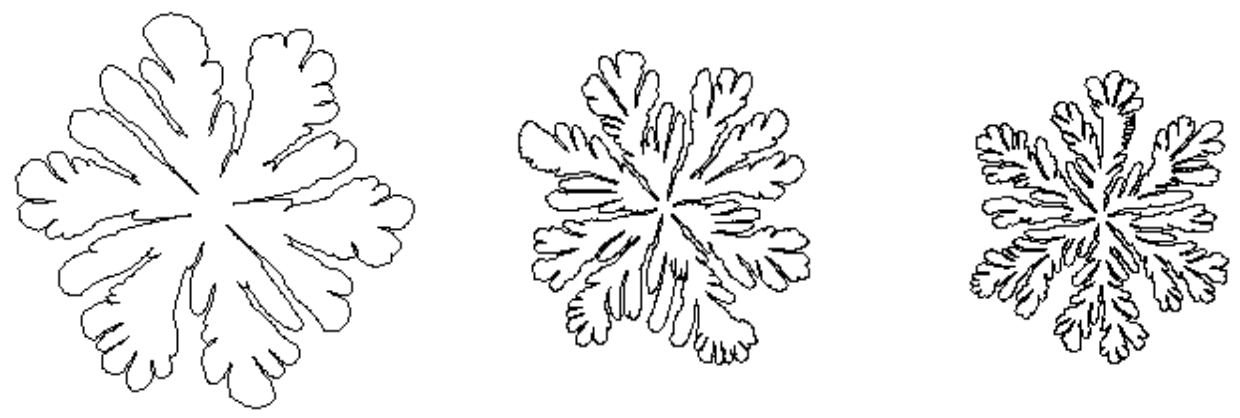

Figure 10: The dendritic structure of precipitation with varying diffusion coefficient $D$ at time $t=0.4$. From left to right: $\alpha=0.1,0.05,0.033$. $D a=500,1000,1500$. The computational grid is $720 \times 720$.

diffusion coefficient represent the cases with higher Damköhler number in solute precipitation. The results show that in higher Damköhler number, the solute precipitation rate is higher compared to the diffusion rate, which causes more dendritic interface in growth process.

\subsection{Precipitation of Single Seed in Flow}

When fluid flow is added to the equation for solute (Eq. (2)), there is an advection effect on the crystal growth. Depending on the direction in which the fluid flows, the growth of precipitation interface is biased. Figure 11 shows the simulation of $2 \mathrm{D}$ precipitation within flowing fluid. In this case, the crystal grows from a seed in a domain $(x, y) \in[0,1] \times[0,3]$ with mass density $\rho_{s}=10$. In this simulation, the upper boundary in y direction is a Dirichlet boundary with constant fluid velocity $v_{y}=-5.0$ and constant pressure as 10.0. The lower boundary in y direction is flow-through boundary. Both boundaries in x direction are periodic. The equilibrium concentration is $C_{e}=$ 1.0. And the initial solute concentration in the ambient area is set to $C(t=$ $0)=2.0$. From Figure 11, it can be seen that in this precipitation simulation, the upper part interface facing the flow grows faster and precipitates more dendritically than the lower part of the interface.

A similar 3D simulation is conducted in a domain $(x, y, z) \in[0,1] \times[0,1] \times$ $[0,2]$. The boundaries in $\mathrm{x}$ and $\mathrm{y}$ direction are all Periodic. The upper boundary in z direction is Dirichlet boundary with constant fluid velocity $v_{0}=-2.0$ and constant solute concentration $C_{0}=2$. The lower boundary in $\mathrm{z}$ direction is a flow-through boundary. The precipitation seed is a sphere with radius $r=0.05$ located at the center of the computational domain, and 

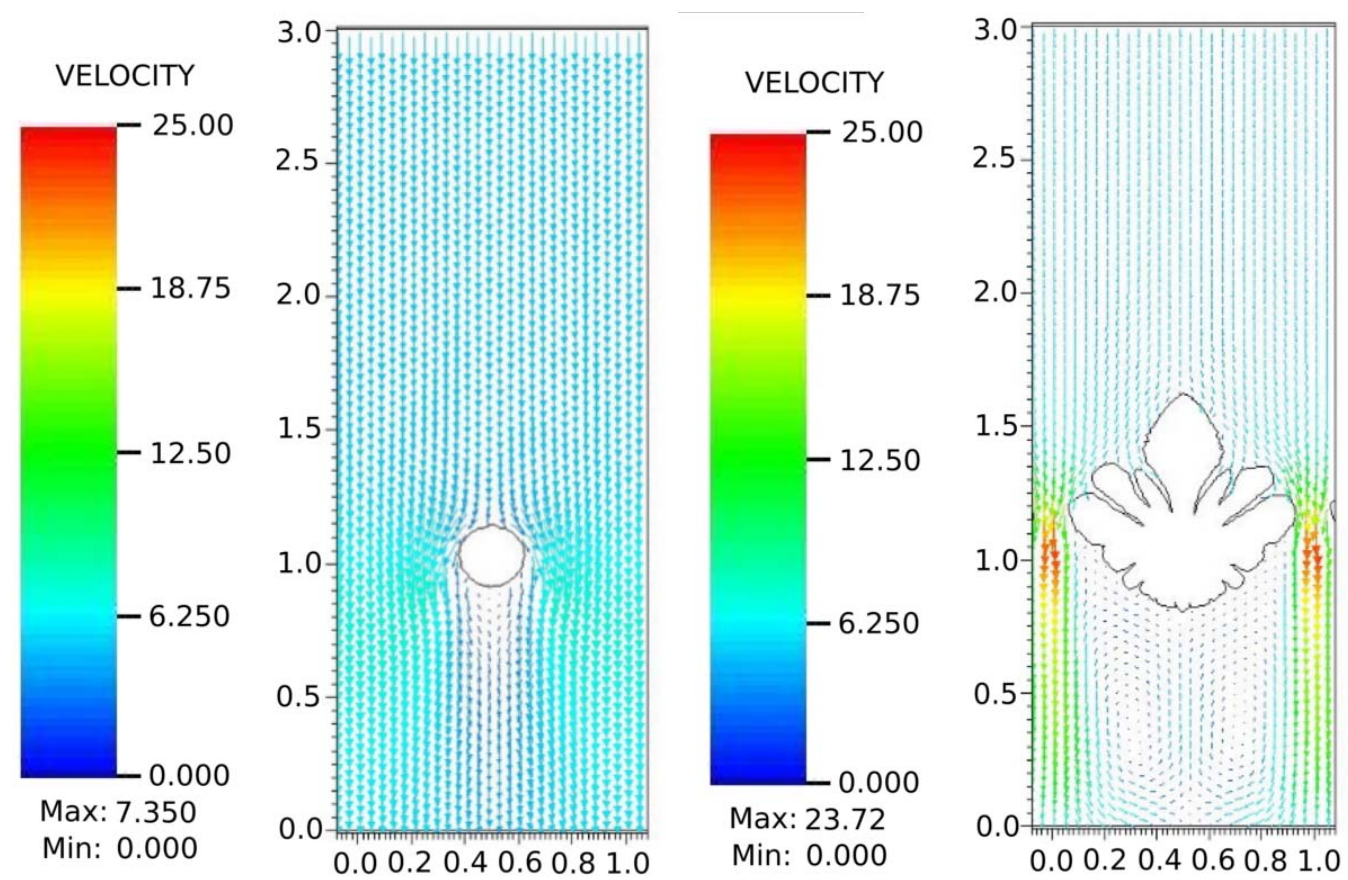

Figure 11: Simulation of precipitation with flowing fluid. The crystal density $\rho_{s}=10$. The equilibrium solubility is $C_{e}=1.0$ at the interface. The initial solute concentration in the ambient area is $C(t=0)=2.0$. The computational grid is $100 \times 300$. 
the crystal density is 10 . The diffusion coefficient $\alpha$ is 0.5 , and the reaction rate $k$ is 80 . The equilibrium solubility $C_{e}$ is 1 , and the initial concentration in the ambient area is 2 . Figure 12 shows the $3 \mathrm{D}$ precipitation interface in fluid flowing from upstream. The upper part grows faster and more dendritic than the lower part.

\subsection{Peclet Number Effect}

The Peclet number $P e=\frac{\mathbf{U}_{\mathbf{c}} L_{c}}{\alpha}$ is a dimensionless parameter reflecting the transport phenomena in flowing fluid. It is the ratio of the advection rate of the fluid flow to the rate of diffusion. We conducted a series of $2 \mathrm{D}$ simulations to study the effect of Peclet number on the precipitation in fluid flow.

The computational domain is $(x, y) \in[0,1] \times[0,4]$. The initial solid interface is a circle with radius $r=0.02$ at the center of the domain. The density of the crystal is $\rho_{s}=10.0$. The reaction rate is $k=80$, and the diffusion coefficient $\alpha=0.5$. The equilibrium concentration in the solution is $C_{e}=1.0$. The initial solute concentration in the ambient fluid is $C(t=0)=$ 2.0. The boundary conditions in $\mathrm{x}$ direction are both Periodic. In $\mathrm{y}$ direction, the lower boundary is flow-through boundary. And the upper boundary in y direction is a Dirichlet boundary with solute concentration $C_{0}=2.0$. The variation of Peclet number is realized by increasing the velocity from the boundary.

Figure 13 shows the precipitation interface with increasing velocity of the flowing fluid. In case of larger Peclet number, the flow advects the solute away from the growing dendrite more quickly, and more solute is condensed on the interface, which results in a faster growth of the crystal interface.

\subsection{Precipitation of Multiple Seeds in Flow}

The crystal growth exhibits more complex pattern when there are multiple seeds. Figure 14 shows the 3D precipitation interface of three sphere seeds located in the computational domain of $(x, y, z) \in[0,1] \times[0,1] \times[0,2]$. In this simulation, we have $\rho_{s}=10.0, k=80$, and $\alpha=0.5$. The equilibrium solubility in the solution is $C_{e}=1.0$. And the initial solute concentration in the ambient area is $C(t=0)=2.0$. The boundary conditions in $\mathrm{x}$ and y directions are both Periodic. In z direction, the lower boundary is a flow-through boundary. And the upper boundary in z direction is Dirichlet boundary with constant solute concentration $C_{0}=2.0$, and constant velocity $v_{0}=-2.0$. 


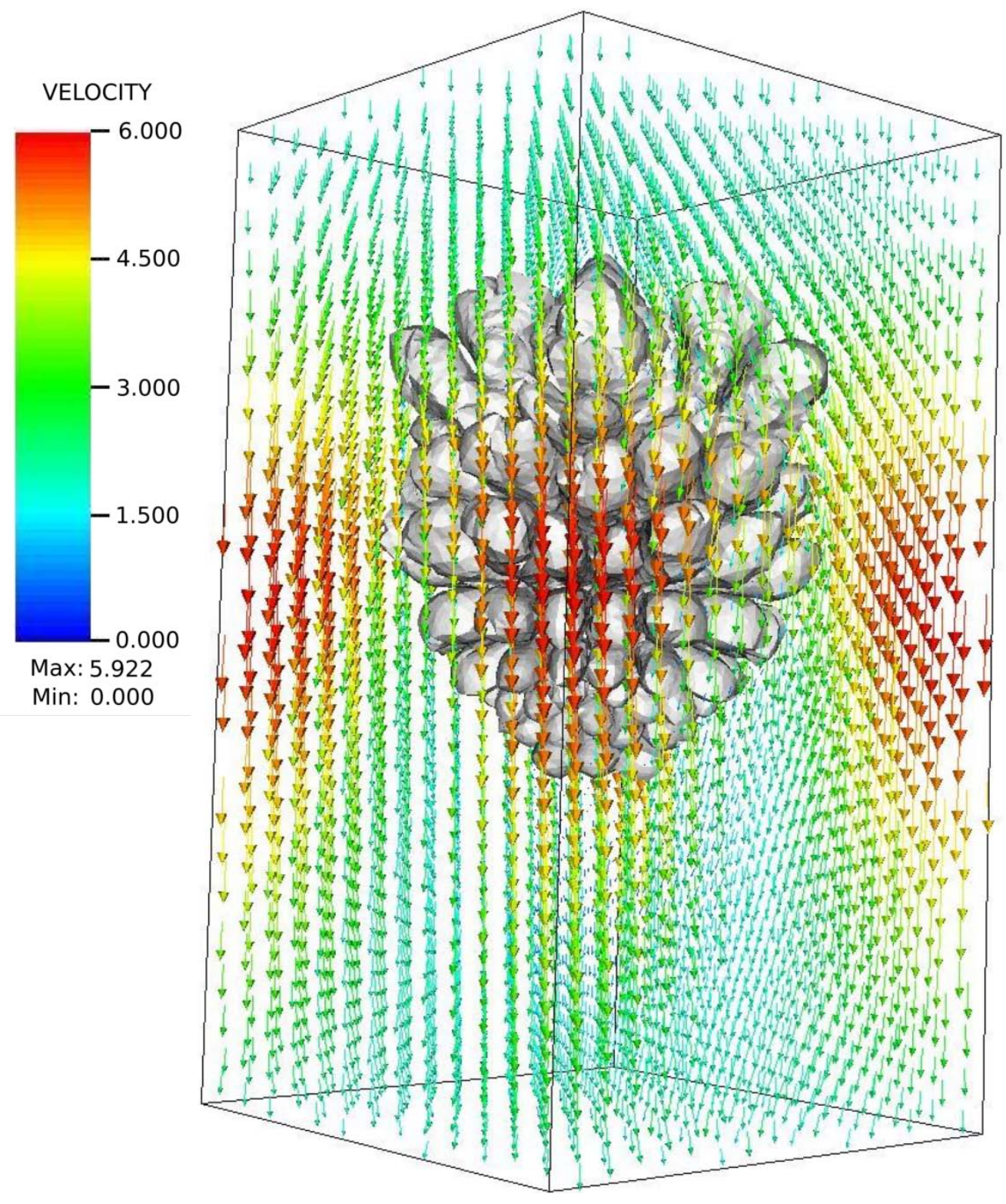

Figure 12: Simulation of 3D precipitation with flowing fluid. The mass density of the solid is $\rho_{s}=10$. The initial ambient concentration is $C(t=0)=2.0$. The equilibrium solubility is $C_{e}=1.0$. The diffusion coefficient is $\alpha=0.5$ and the reaction rate is $k=80$. The computational grid is $60 \times 60 \times 120$. 

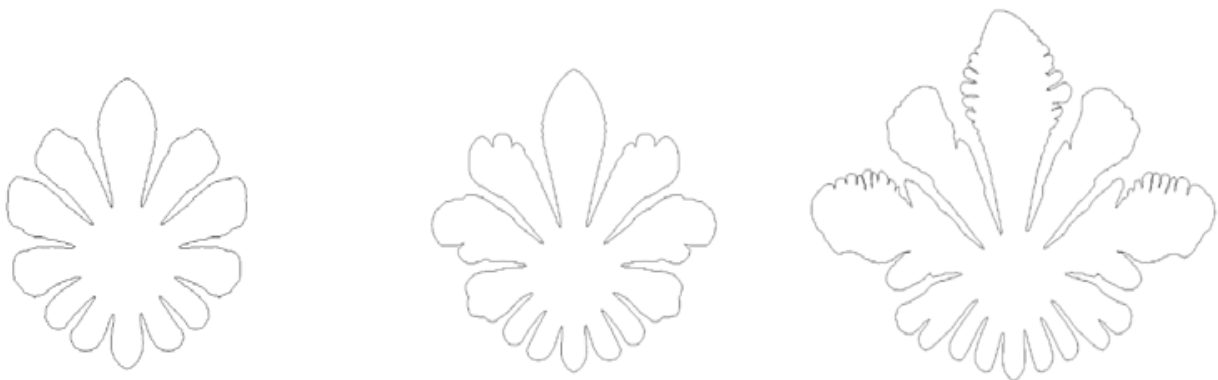

Figure 13: The interface of precipitation with varying flowing fluid velocity at time $t=$ 0.55 . From left to right, the flowing fluid velocity is: $\{0,-2\},\{0,-4\},\{0,-8\}$. The computational grid is $100 \times 400$.

Since the fluid flow is from the upper part, the upper side of top seed facing the flow grows faster than the lower side and the seeds below it. The growth between two seeds stops when their interfaces become closer to each other. The fluid flow has almost no effect in the space between the two seeds.

\section{Numerical Results for Dissolution}

Numerical simulations of dissolution were carried out in two dimension on both homogeneous and non-homogeneous materials, as well as on different initial interface geometry. We measured the variation of solute concentration and surface area in the dissolution process. We define the dissolution ratio as follows,

$$
\bar{\xi}=\frac{P}{S},
$$

where $P$ is the perimeter, and $S$ is the area of the dissolving solid. The significance of this ratio is that it reflects the average material dissolved on a given length of the interaction surface (perimeter in 2D). The area of the material is calculated using the grid-based cut-cell method.

\subsection{Dissolution of a Slotted Circle}

For dissolution of a slotted circle, the computational domain is $(x, y) \in$ $[0,6] \times[0,6]$. The initial interface is a circle centered at $(3.0,3.0)$ with radius as 0.5 . The four slots are perpendicular to each other. The width and depth of each slot are 0.1 and 0.42 , respectively. One vertical slot is facing the fluid flow, one is at the opposite side of the flow, and two horizontal slots 


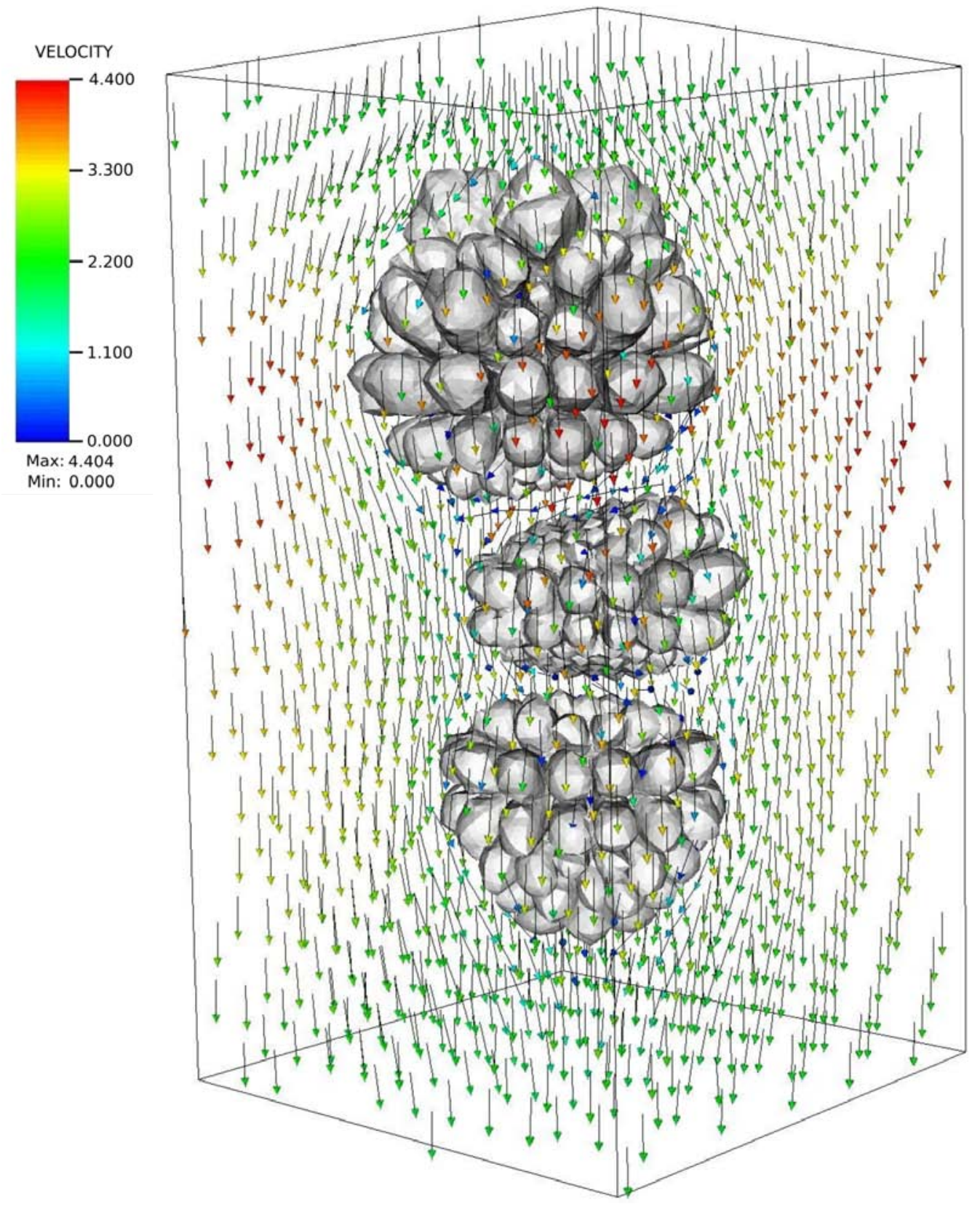

Figure 14: Precipitation of multiple seeds with flowing fluid in 3D. The mass density of the solid is $\rho_{s}=10.0$. The reaction rate is $k=80$, and the diffusion coefficient is $D=0.5$. The equilibrium solubility is $C_{e}=1.0$. And the initial solute concentration in the ambient area is $C(t=0)=2.0$. The computational grid is $60 \times 60 \times 120$. 

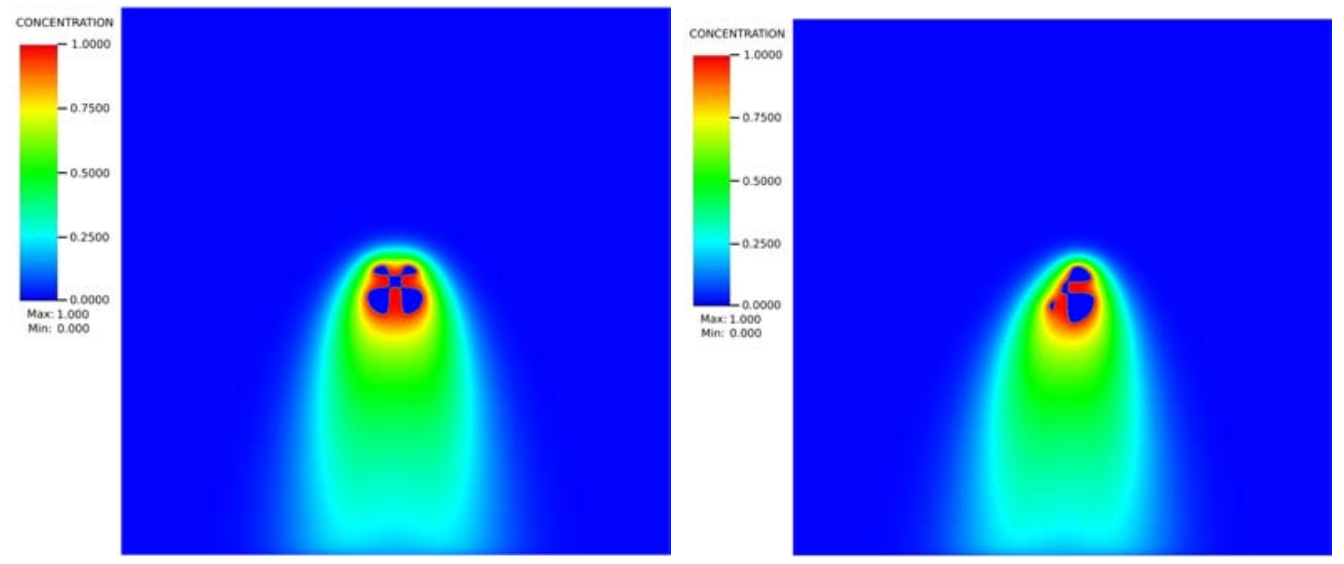

Figure 15: 2D dissolution of homogeneous and non-homogeneous four-slotted circle. The figure shows the dissolving interface and solute concentration at time $t=3.2$. The diffusion coefficient is $\alpha=0.1$, and the reaction rate is $k=500$. The equilibrium solubility is $C_{e}=$ 1.0. And the initial condition in the ambient area is $C(t=0)=0.0$. The computational grid is $800 \times 800$.

are normal to the flow. This simulation is designed to study the dissolution rate at both the convex and concave parts of the material, and the advection effects on the concavity of the material.

The diffusion coefficient in the simulation is $\alpha=0.1$, the reaction rate is $k=500$, and the equilibrium solubility is $C_{e}=1.0$. The initial condition in the ambient concentration is set to be $C(t=0)=0.0$. For boundary conditions, we set both lower and upper boundaries in $\mathrm{x}$ direction as periodic. The upper boundary in y direction is Dirichlet boundary with constant solute concentration $C_{0}=0$ and velocity $\mathbf{v}_{0}=(0,-2.0)$, and the lower boundary in $\mathrm{y}$ direction is flow-through.

For the homogeneous case, the mass density of the dissolving material is 10. And for the non-homogeneous case, we set the mass density as 5 for the left side $x<3.0$ and 15 for the right side $x>3.0$. Figure 15 shows the dissolving interface of the homogeneous and non-homogeneous solid material and solute concentration (in color) at time $t=3.2$.

The region close to the dissolving interface has a much higher solute concentration than the other places in the liquid phase. Especially near the concave part of the slotted interface, the solute concentration is almost equal to the equilibrium solubility $C_{e}$. The dissolution in these parts are very 


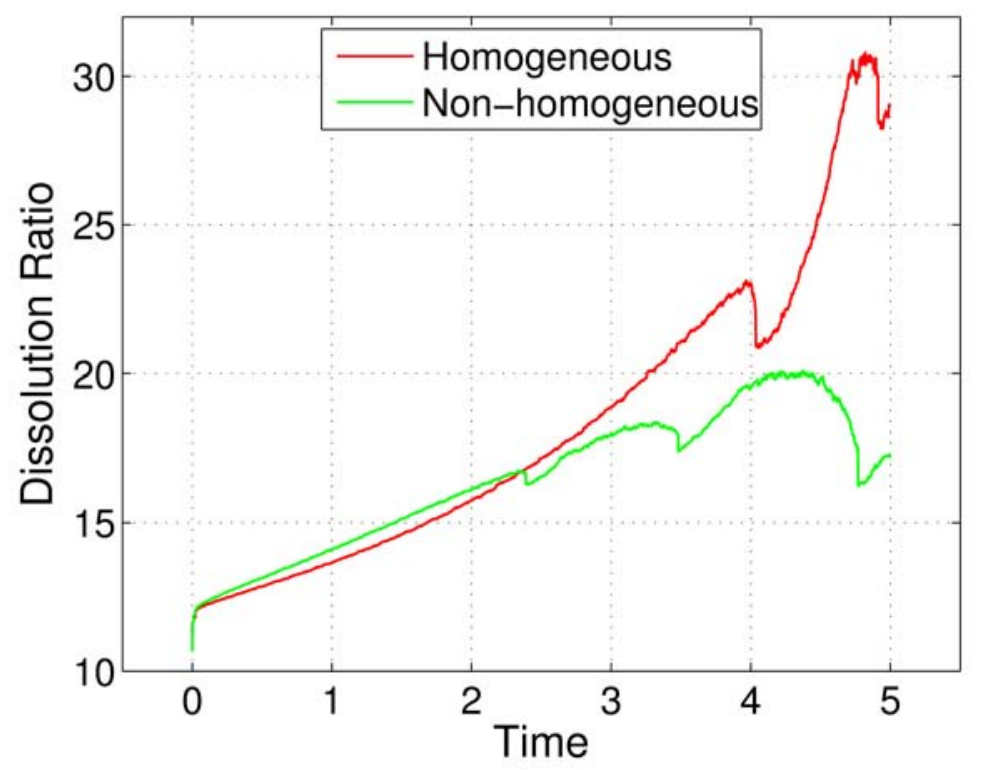

Figure 16: 2D dissolution of homogeneous and non-homogeneous four-slotted circle. It shows the changes of dissolution ratio in two simulations.

slow. Due to the advection of fluid flow, the solute at the upper part of the interface is flushed away quickly than that in the lower part. As a result, the rate of dissolution in upper part is much faster than that in the lower part. Therefore, there is a bias from upper part to the lower part for dissolving material. In homogeneous case, the interface is almost symmetric about the center line $x=3.0$, while in the non-homogeneous case, the interface of the left side dissolves faster than the right side due to the smaller density of the dissolving material in the left side.

The red curve in Figure 16 shows the changes of dissolution ratio during the dissolution process of the homogeneous material. The dissolution ratio measures material at the dissolving front vs. the material as a whole. In general, this ratio increases as the average curvature of the boundary decreases. The initial stage of the dissolution ratio curve is consistent with this rule. The first sudden drop of the red curve in Figure 16 around time $t=4.0$ is due to the full dissolution and disappearance of the two upper solid break-ups. The second drop of the curve corresponds to the complete dissolution of the central piece. 
Like the homogeneous case, in the non-homogeneous case, the dissolution in the upper part is faster than that of the lower part due to the advection effect of the fluid flow. However, unlike the homogeneous case, the left side dissolves faster than the right side due to the density difference of the material. The disk dissolves into pieces and then each piece dissolves completely at different time. First, the upper-left piece, then the central piece, etc. The disappearance of each piece causes a drop in the dissolution ratio curve, as shown by the green curve in Figure 16.

\subsection{Dissolution of Porous Particle}

We set a perturbed circle initialization with non-homogeneous mass density to mimic the porous particle. The computational domain is $(x, y) \in$ $[0,4] \times[0,5]$. The initial interface is a perturbed circle centered at $(2.0,3.0)$ with radius defined as $r=0.5-0.01 \sin \left(24 \theta+\frac{3}{2} \pi\right)$. The diffusion coefficient is $\alpha=0.1$, the reaction rate is $k=400$, and the equilibrium solubility $C_{e}$ is 1 . We place the dissolving material in a fluid with density and viscosity as 0.1 and 0.00628 . The initial condition in the ambient area is set to be $C(t=0)=0.0$. For boundary conditions, both lower and upper boundaries in $\mathrm{x}$ direction are Periodic. The upper boundary in $\mathrm{y}$ direction is Dirichlet boundary with solute concentration as 0 and fluid velocity $\mathbf{v}_{0}=(0,-2)$. The lower boundary in y direction is a flow-through boundary.

The non-homogeneous solid density has porosity $13.1 \%$, with pores randomly distributed inside the solid, as shown by the upper-left plot of Figure 17. The upper-right plot of Figure 17 shows the evolution of the interface during the dissolution process. The lower plot shows the solute concentration field around the interface at time $t=3.2$.

We also carried out a simulation of dissolution of a composite material to mimic the dissolution of metal oxide in nitride acid. The initial geometry and density of the material is provided by the Oak Ridge National Lab and read into the FronTier code. The acid is in the liquid phase with metalsolute density zero. The dissolving material mimic the metal oxide with two different density values $\rho_{1}=1.2$ and $\rho_{2}=6.0$ respectively. Here the computational domain is set in $(x, y) \in[-24.415,73.245] \times[-24.415,73.245]$. The diffusion coefficient is $\alpha=0.1$, the reaction rate is $k=500$, and the equilibrium solubility $C_{e}$ is 1 . The initial condition in the ambient area is set to be $C(t=0)=0.0$. For the boundary conditions, we set both lower and upper boundaries in $\mathrm{x}$ direction as Periodic. The upper boundary in y direction is Dirichlet boundary with solute concentration as 0 and velocity of the fluid as 

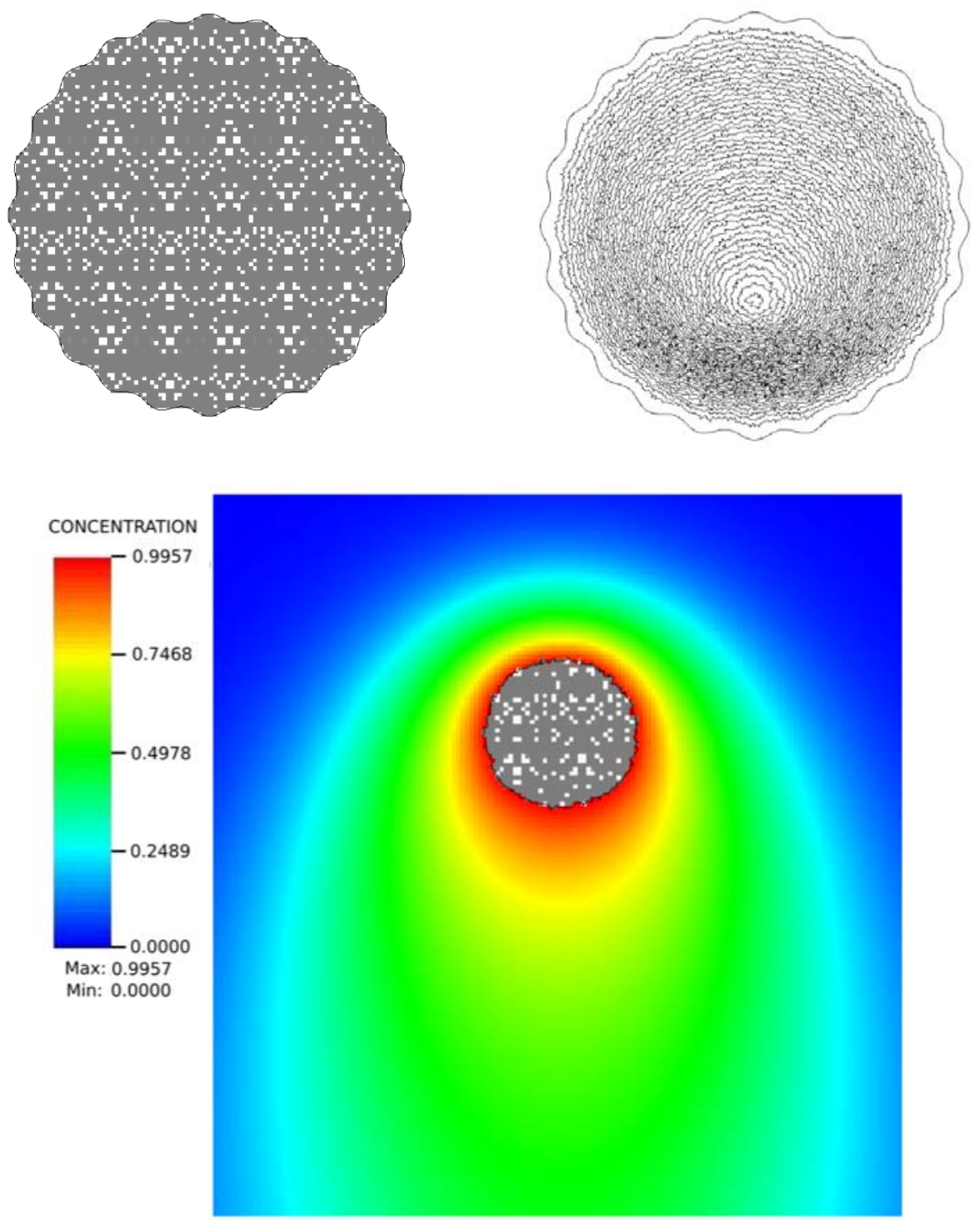

Figure 17: 2D dissolution of porous particle. The upper left plot shows the nonhomogeneous initial mass density of the dissolving material. The upper right plot shows the evolution of dissolving interface. The lower plot shows the solute concentration field around interface at time $t=3.2$. The diffusion coefficient is $\alpha=0.1$, the reaction rate is $k=400$. The computational grid is $400 \times 500$. 
$\mathbf{v}_{0}=(0,-2)$, the lower side in y direction is a flow-through boundary. The left plot Figure 18 shows the pore structure of the sample metal oxide and right plot shows the corresponding simulation domain. Figure 19 shows the erosion of the metal oxide in the convecting solvent.
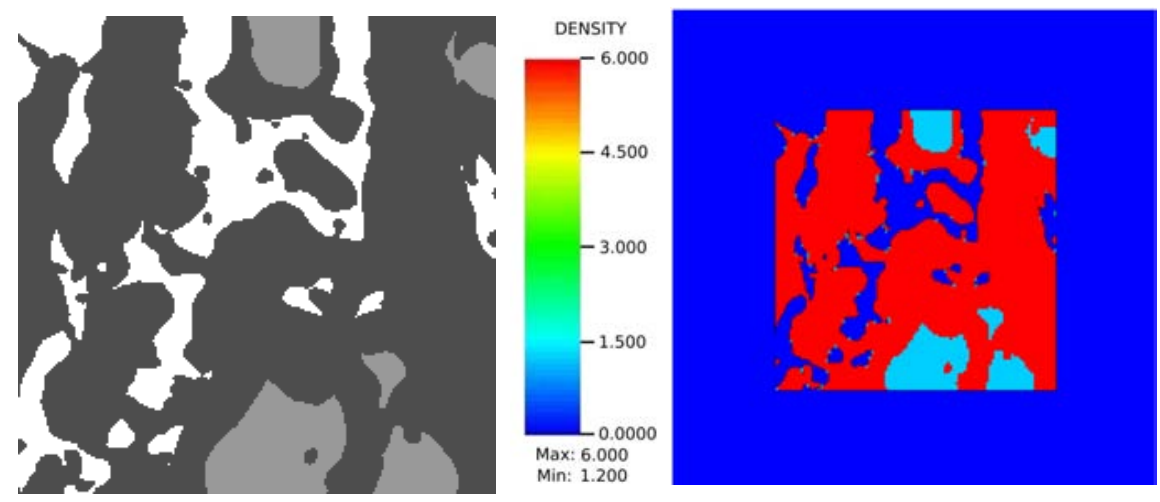

Figure 18: 2D dissolution of a composite material. The figure shows the initial density and the corresponding interface of the metal oxide. The diffusion coefficient is $\alpha=0.1$, and the reaction rate $k=500$. The equilibrium solubility is $C_{e}=1.0$. And the initial condition in the ambient area is $C(t=0)=0$. The computational grid is $200 \times 200$.

This simulation demonstrates the capability of the FronTier code in handling dissolution and advection on Heterogeneous material. Such capability can be applied to engineering simulations such as the nuclear waste disposal problem.

\section{Numerical Results for Melting}

For 2D cases, we apply our numerical simulation system into ice-water phase transition problems. For this problem, we use realistic values of physical parameters for both water and ice. Table 1 shows the physical values of thermal conductivity, specific heat, and density for ice and water in different temperature [50, 51].

We study the phase transition process of a small ice particle with temperature $233.15 \mathrm{~K}$ in the water with temperature $283.15 \mathrm{~K}$. The computational domain is $(x, y) \in[0,0.1] \times[0,0.1]$. The initial solid interface is a perturbed circle centered at $(0.05,0.05)$ with radius defined as $r=$ $0.015-0.003 \sin \left(6 \theta+\frac{1}{18} \pi\right)$, which stands for a small rough ice particle with radius around $1.5 \mathrm{~cm}$. The thermal parameters for the ice and water are 

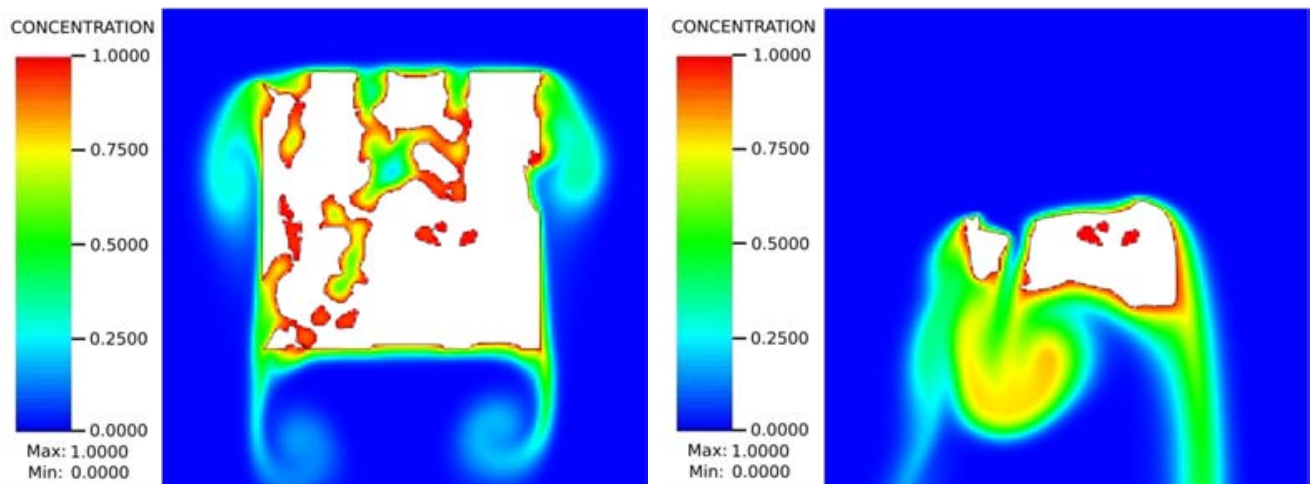

Figure 19: 2D dissolution of a composite material. The figure shows the dissolving interface, and the field of solute concentration around the dissolving interface at time $t=0.25$ minutes (left), and 20.583 minutes (right), respectively.

\begin{tabular}{ccccc}
\hline Material & Temperature & Thermal Conductivity & Specific Heat & Density \\
\cline { 2 - 5 } & $K$ & $W \cdot \mathrm{m}^{-1} \cdot \mathrm{K}^{-1}$ & $\mathrm{~J} \cdot \mathrm{kg}^{-1} \cdot \mathrm{K}^{-1}$ & $\mathrm{~kg} \cdot \mathrm{m}^{-3}$ \\
\hline Ice & 233.15 & 2.63 & $1.818 \times 10^{3}$ & 920.8 \\
\hline Water & 283.15 & 0.580 & $4.193 \times 10^{3}$ & 999.8 \\
\hline
\end{tabular}

Table 1: Values for physical parameters in melting process 
shown in Table 1. The latent heat for this ice-water transition simulation is $3.34 \times 10^{5} \mathrm{~J} \cdot \mathrm{kg}^{-1}$. For boundary conditions, we set both lower and upper boundaries in $\mathrm{x}$ and $\mathrm{y}$ direction as Neumann boundaries to mimic the heat-insulated domain. We simulate this problem by implicit scheme in a $160 \times 160$ computational grid.

Figure 20 shows the ice-water interface and the temperature field. The diffusion coefficient in the ice is about 11 times of that in the water. Therefore, the temperature in the ice is equalized quickly, which is $273.15 \mathrm{~K}$. At a later time, the temperature in the water also reaches equilibrium. Figure 21 shows the changes of temperature in a sample line of $y=0.05$ at time $t=0.405$ minutes, 7.169 minutes, 68.79 minutes, 168.48 minutes. Since the whole system is heat-insulated and the ice is heated by the water during melting process, the temperature at the boundary is decreased gradually. Eventually, the temperature in the entire computational domain reaches equilibrium. This is shown by the comparison of temperature profile at time $t=$ 0 s and time $t=168.48$ minutes in Figure 22 .

We also conducted the 3D ice-water transition between $233.15 K$ ice cube and the $283.15 K$ water. The physical properties are shown in Table 1 . The computational domain is $(x, y, z) \in[0,0.1] \times[0,0.1] \times[0,0.1]$. The initial shape of ice is cubic with length as $0.03 \mathrm{~m}, 0.02 \mathrm{~m}, 0.024 \mathrm{~m}$ centered at $(0.05,0.05,0.05)$. The boundary in each direction is Neumann boundary to mimic the heat-insulated environment.

Figure 23 shows the melting interface of the ice cube at time $t=0$, 26.67minutes, 53.33 minutes, 80 minutes respectively. Figure 24 shows the temperature field in the simulation. Since the system is heat-insulated and the ice is heated by the water, the temperature in the water decreases gradually.

\section{Conclusions}

Phase transition process is widely used in chemical, physical and industrial field. The main objective of the present work is to develop a robust numerical algorithm and computational platform for the study of these phase transition problems. In this paper, we presented the front tracking method for the simulations of two and three dimensional phase transition problems. This includes precipitation, dissolution, freezing and melting problems. Using the front tracking data structure and functionality, we can track the phase transition interface accurately in both $2 \mathrm{D}$ and $3 \mathrm{D}$ with complex and changing 

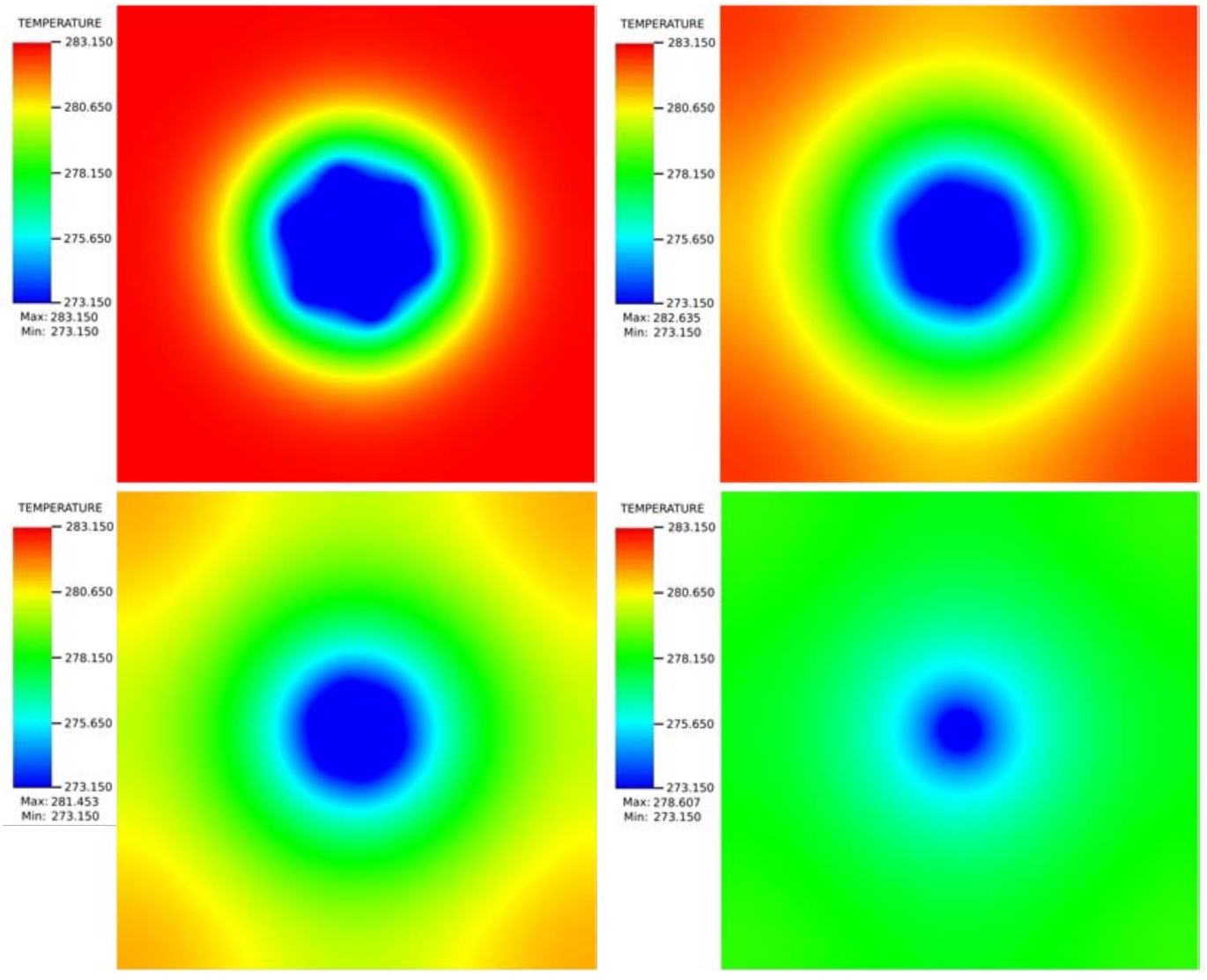

Figure 20: 2D melting of $233.15 \mathrm{~K}$ ice particle in $283.15 \mathrm{~K}$ water at time $t=9.167$ minutes, 36.67 minutes, 64.167 minutes, 155.83 minutes from left to right. The thermal conductivity of ice is $2.63 \mathrm{~W} \cdot \mathrm{m}^{-1} \cdot \mathrm{K}^{-1}$. The specific heat of ice is $1.818 \times 10^{3} \mathrm{~J} \mathrm{~kg}^{-1} \cdot \mathrm{K}^{-1}$. And the density of ice is $920.8 \mathrm{~kg} \cdot \mathrm{m}^{-3}$. The thermal conductivity of water is $0.580 \mathrm{~W} \cdot \mathrm{m}^{-1} \cdot \mathrm{K}^{-1}$. The specific heat of water is $4.193 \times 10^{3} \mathrm{~J} \mathrm{~kg}^{-1} \cdot \mathrm{K}^{-1}$. And the density of water is $999.8 \mathrm{~kg} \cdot \mathrm{m}^{-3}$. 


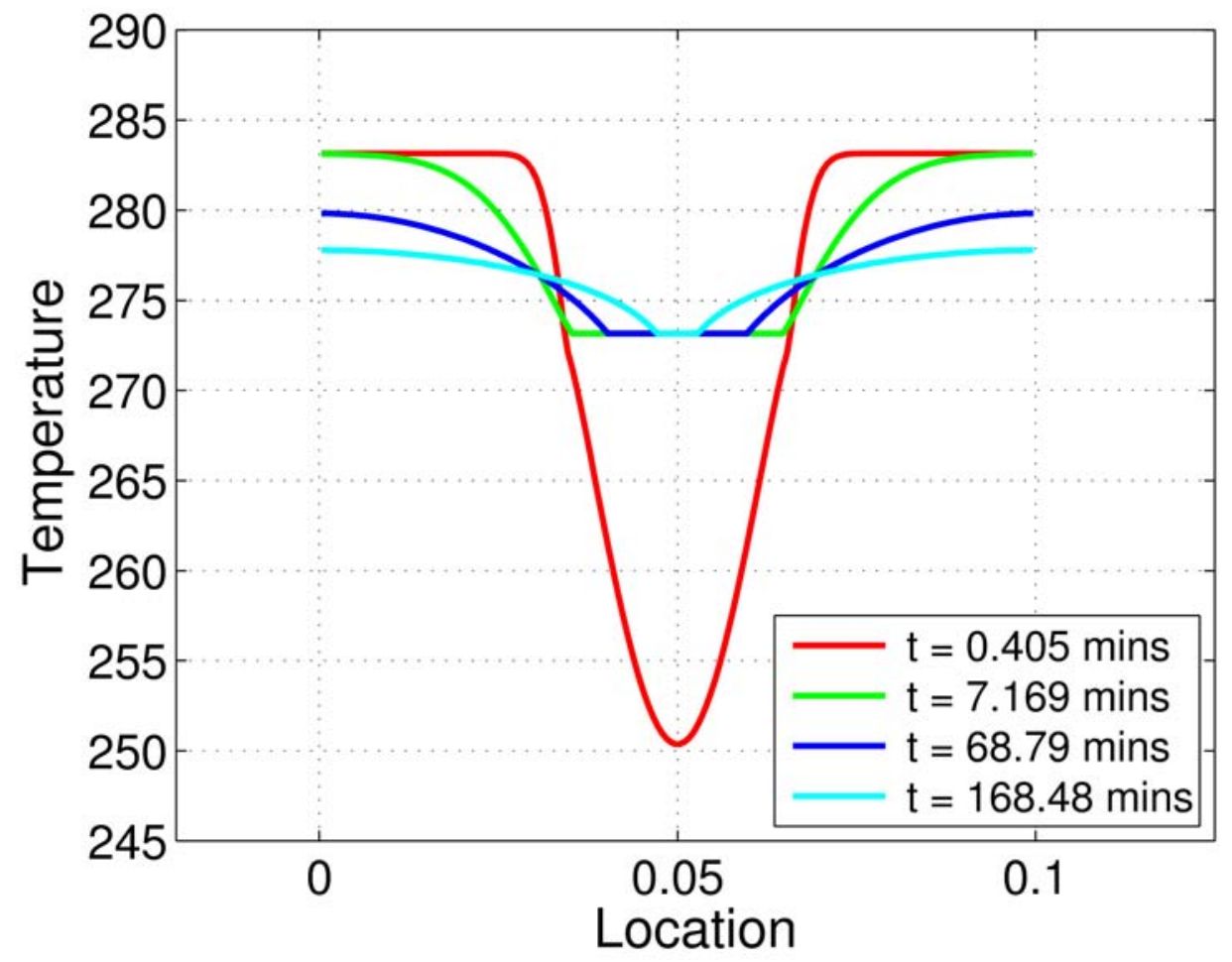

Figure 21: 2D melting of $233.15 K$ ice particle in the $283.15 K$ water. It shows temperature in the sample line $y=0.05$ at time $t=0.405$ minutes, 7.169 minutes, 68.79 minutes, 168.48 minutes. 


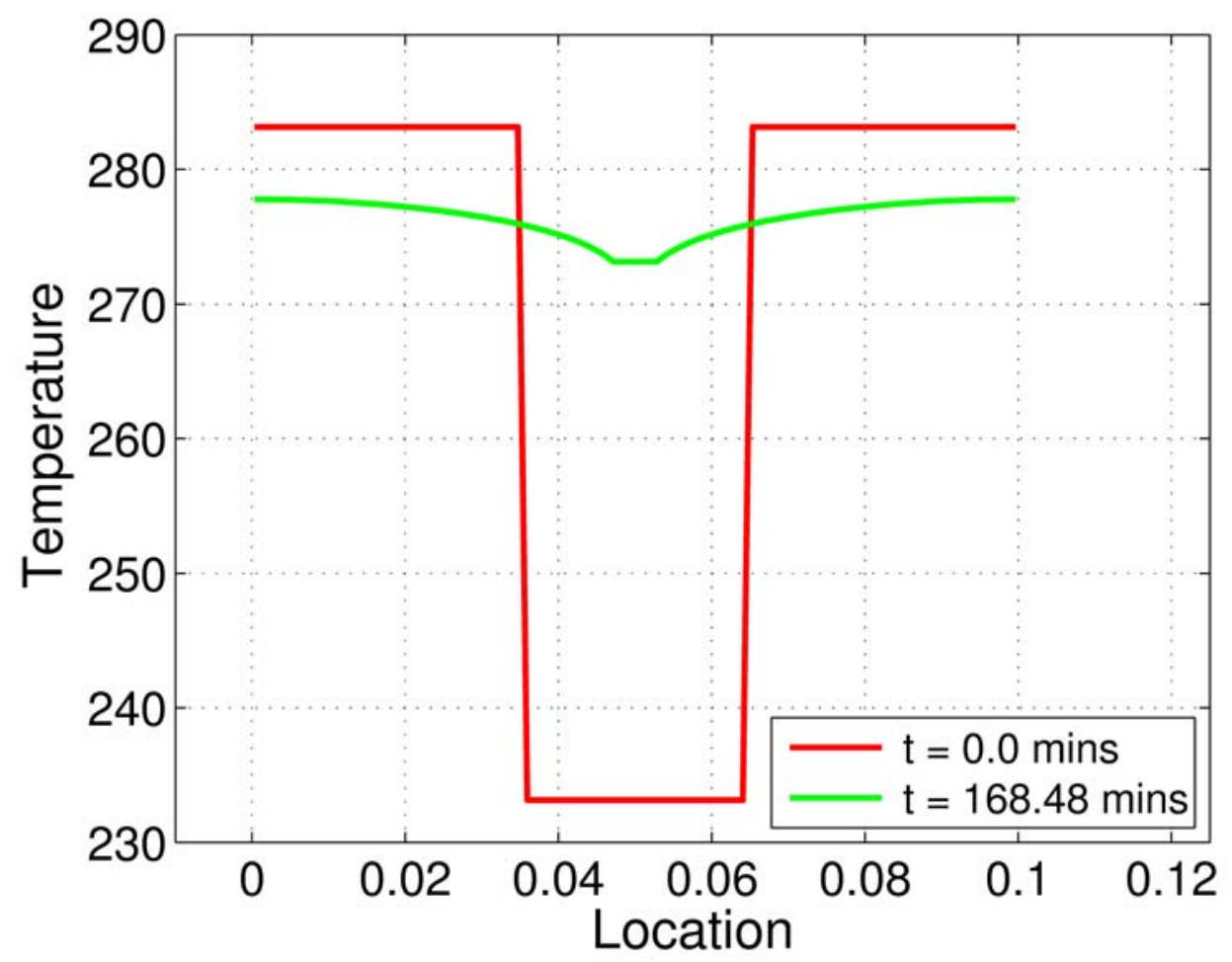

Figure 22: 2D melting of ice particle $233.15 K$ in the $283.15 K$ water. The red line shows the temperature in the sample line $y=0.05$ at time $t=0 \mathrm{~s}$. And the green line shows the temperature in the sample line $y=0.05$ at time $t=168.48$ minutes. 

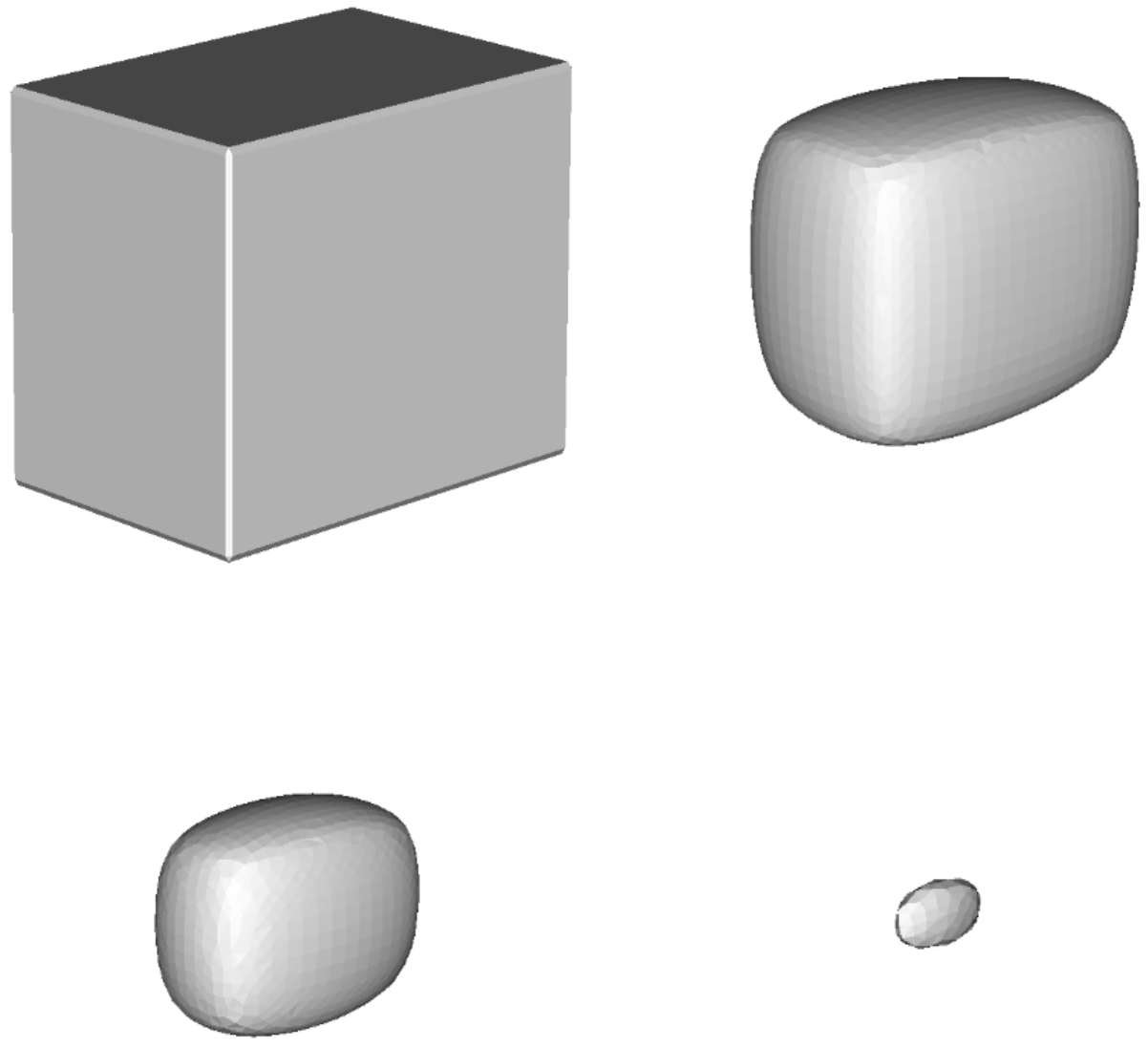

Figure 23: The 3D melting interface of $233.15 \mathrm{~K}$ ice cube in the $283.15 \mathrm{~K}$ water at time $t=0$ minute, 26.67 minute, 53.33 minutes, 80 minutes from left to right. The thermal conductivity of ice is $2.63 \mathrm{~W} \cdot \mathrm{m}^{-1} \cdot \mathrm{K}^{-1}$. The specific heat of ice is $1.818 \times 10^{3} \mathrm{~J} \cdot \mathrm{kg}^{-1}$. $K^{-1}$. And the density of ice is $920.8 \mathrm{~kg} \cdot \mathrm{m}^{-3}$. The thermal conductivity of water is $0.580 \mathrm{~W} \cdot \mathrm{m}^{-1} \cdot \mathrm{K}^{-1}$. The specific heat of water is $4.193 \times 10^{3} \mathrm{~J} \cdot \mathrm{kg}^{-1} \cdot \mathrm{K}^{-1}$. And the density of water is $999.8 \mathrm{~kg} \cdot \mathrm{m}^{-3}$. 

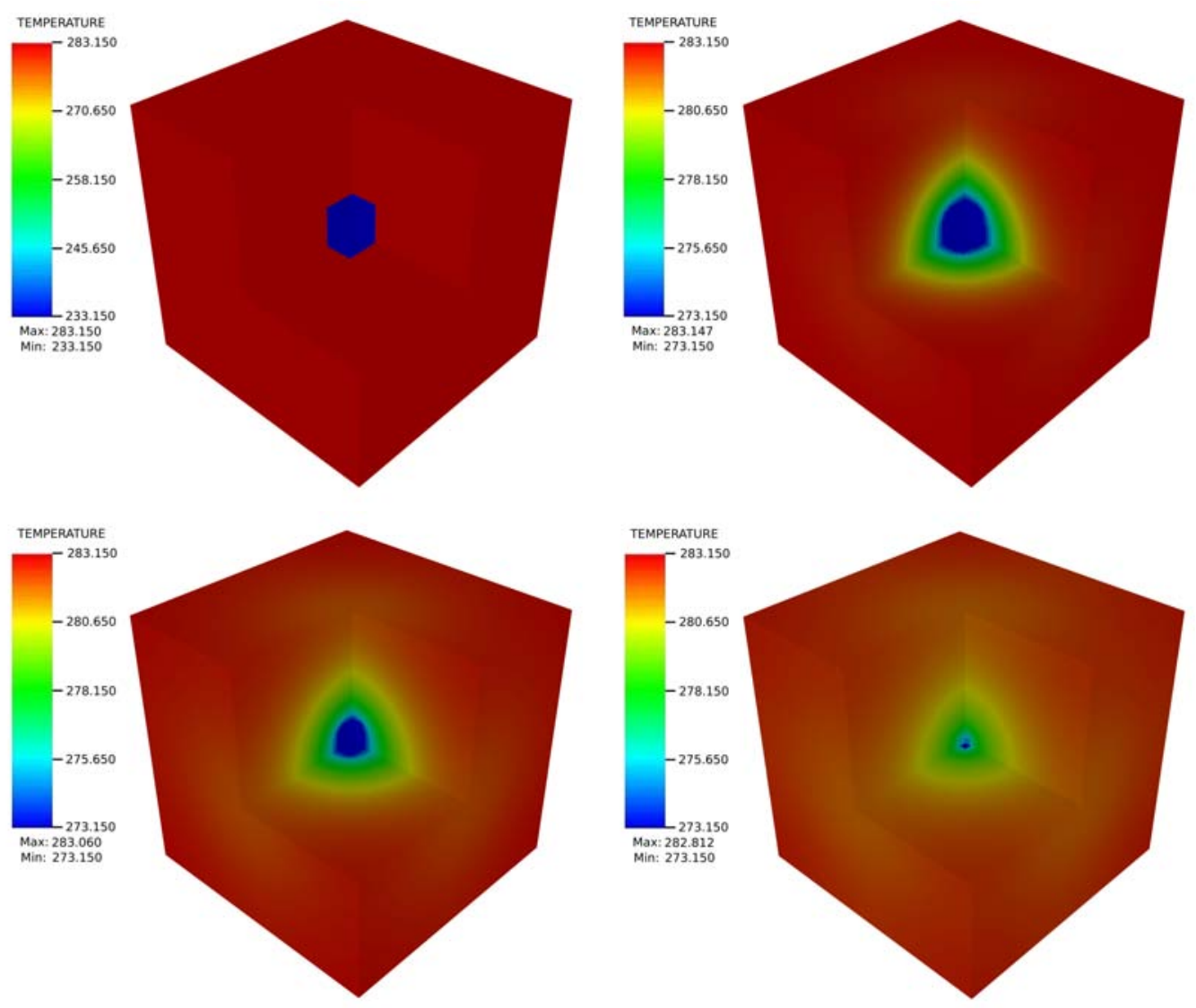

Figure 24: The 3D temperature field of $233.15 \mathrm{~K}$ ice cube in the $283.15 \mathrm{~K}$ water at time $t=0,26.67$ minutes, 53.33 minutes, 80 minutes from left to right. The thermal conductivity of ice is $2.63 \mathrm{~W} \cdot \mathrm{m}^{-1} \cdot \mathrm{K}^{-1}$. The specific heat of ice is $1.818 \times 10^{3} \mathrm{~J} \cdot \mathrm{kg}^{-1} \cdot \mathrm{K}^{-1}$. And the density of ice is $920.8 \mathrm{~kg} \cdot \mathrm{m}^{-3}$. The thermal conductivity of water is $0.580 \mathrm{~W} \cdot \mathrm{m}^{-1} \cdot \mathrm{K}^{-1}$. The specific heat of water is $4.193 \times 10^{3} \mathrm{~J} \mathrm{~kg}^{-1} \cdot \mathrm{K}^{-1}$. And the density of water is $999.8 \mathrm{~kg} \cdot \mathrm{m}^{-3}$. 
interface geometry and topology. We use finite difference schemes to solve the underlying PDE in each subdomain and propagate the phase transition interface using conservation laws of mass or energy. The phase transition solver is coupled with incompressible Navier-Stokes solver to mimic the advection effect.

The simulations of precipitation show the explicitly tracked interface of crystal growth. With higher Damköhler number, the precipitation interface is more dendritic than the interface in lower case. With the inclusion of advection by fluid flow, there is bias in the precipitation interface. The area facing to the flowing fluid grows faster and more dendritic, while the other part tends to grow more slowly. For the dissolution in fluid flow, we analyzed the dissolution ratio of the dissolving solid. The dissolution rate is dependent on the mass density of the dissolving material. Therefore, the part of material with lower density dissolves faster than the part with higher density. In general the interface is smoothed in the dissolution process, but for a porous particle, the interface could still be roughened due to the anisotropy of the density distribution. Advection has strong effect on dissolution too. The side facing the fluid flow dissolves much faster than the side in the downstream of the flow. Using different conservation law at the interface (conservation of heat), we studied the melting and the ice-water phase transition process in both two and three dimensions.

The phase transition problem is a complex system. A high order simulation would require that each component of the system has the same order of accuracy together with the compatible order in the coupling of these computational components. We have used second order PDE solvers for both diffusion and advection equations, we have also used second order method in computation of geometrical variables such as normal and curvature. But we have not achieved overall second order due to lower order coupling of these components, especially when the interface geometry undergoes topological changes. For further improvement of this system, we plan to use the Coupling Interface Method (CIM) by Chern and Shu 52] to enhance the accuracy of the system. The Coupling Interface Method features dimensionally splitting algorithm in the Cartesian grid and is an ideal method for our system. We plan to present the coupling with CIM in our next paper.

At the application front, we would like to apply our numerical system on different types of phase transition applications and make comparison with experimental results. Such applications include climate modeling and weather prediction. We are particularly interested in the study of freezing and evapo- 
ration of the cloud droplet and its size distribution, and the comparison with experiments such as the one by Natasha L. Miles et al. [53].

\section{Acknowledgment}

This work was sponsored by the U.S. Department of Energy through the Office of Nuclear Energy in the Nuclear Energy Advanced Modeling and Simulation project, under contract DE-AC05-00OR22725 with UT-Battelle, LLC. Xiaolin Li was supported in part by the US Army Research Office under the ARO grant award W911NF0910306. Yijing Hu was support in her last academic year by the US Army Research Office under the ARO grant award W911NF0910306.

\section{References}

[1] C. Chang, D. Flamm, D.E.Ibbostson, J.A.Mucha, Diamond crystsal growth by plasma chemical vapor deposition, Journal of Applied Physics 63 (1988) 1744-1748.

[2] L. Besra, M. Liu, A review on fundamentals and applications of electrophoretic deposition (epd), Progress in Materials Science 52 (1-61) 2007.

[3] L. R. Koenig, Numerical modeling of ice deposition, Journal of the Atmospheric Sciences 28 (1970) 226-237.

[4] P. B. Kelemen, J. A. Whitehead, E. Aharonov, K. A. Jordahl, Experiments on flow focusing in soluble porous media, with applications to melt extraction from the mantle, Journal of Geophysical Research 100 (1995) 475-496.

[5] E. Aharonov, J. A. Whitehead, P. B. Kelemen, M. Spiegelman, Channeling instability of upwelling melt in the mantle, Journal of Geophysical Research 100 (1995) 20,433-20,450.

[6] E. Aharonov, M. Spiegelman, P. Kelemen, Three-dimensional flow and reaction in porous media: Implications for the earth's mantle and sedimentary basins, Journal of Geophysical Research 102 (1997) 14,82114,833 . 
[7] P. Ortoleva, J. Chadam, E. Merino, A. Sen, Geochemical selforganization ii: the reactive-infiltration instability, American Journal of Science 287 (1987) 1008-1040.

[8] J. F. McCarthy, J. M. Zachara, Subsurface transport of contaminants, Environ. Sci. Technol. 23 (1989) 496-502.

[9] S. E. Powers, L. M. Abriola, W. J. W. JR, An experimental investigation of nonaqueous phase liquid dissolution in saturated subsurface systems: Steady state mass transfer rates, Water Resources Research 28 (1992) 2691-2705.

[10] M. J. Oldham, R. F. Phalen, T. Heistracher, Computational fluid dynamic predictions and experimental results for particle deposition in an airway model, Aerosol Science and Technology 32 (2000) 61-71.

[11] H. Hartmann, J.J.Derksen, H. den Akker, Numerical simulation of a dissolution process in a stirred tank reactor, Chemical Engineering Science 61 (2006) 3025-3032.

[12] C. I. Steefel, A. C. Lasaga, A coupled model for transport of multiple chemical species and kinetic precipitation/dissolution reactions with application to reactive flow in single phase hydrothermal systems, American Journal of Science 294 (1994) 529-592.

[13] Q. Kang, D. Zhang, S. Chen, Simulation of dissolution and precipitation in porous media, J. Geophys. Res. 108 (2003) 2505.

[14] Q. Kang, D. Zhang, S. Chen, X. He, Lattice boltzmann simulation of chemical dissolution in porous media, Physical Review E 65 (2002) 036318 .

[15] N. Nagai, S. Miyamoto, M. Nishiwaki, M. Takeuchi, Numerical simulation of snow melting on pavement surface with heat dissipation pipe embedded, Trans JSME 74 (2008) 640-647.

[16] S. Bekri, J. F. Thovert, P. M. Adler, Dissolution of porous media, Chemical Engineering Science 50 (1995) 2765-2791. 
[17] P. Moszkowicz, J. Pousin, F. Sanchez, Diffusion and dissolution in a reactive porous medium: Mathematical modelling and numerical simulations, Journal of Computational and Applied Mathematics 66 (1996) $377-389$.

[18] P. Dijk, B. Berkowitz, Precipitation and dissolution of reactive solutes in fractures, Water Resources Research 34 (1998) 457-470.

[19] M. E. Coltrin, R. J. Kee, J. A. Miller, A mathematical model of the coupled fluid mechanics and chemical kinetics in a chemical vapor deposition reactor, Journal of the Electrochemical Society 131 (1984) 425-434.

[20] C. N. Fredd, H. S. Fogler, Influence of transport and reaction on wormhole formation in porous media, AIChE J. 44 (1998) 1933-1949.

[21] M. L. Hoefner, H. S. Fogler, Pore evolution and channel formation during flow ad reaction in porous media, AIChE J. 34 (1988) 45-54.

[22] M. Farzaneh, K. Savadjiev, Statistical analysis of field data for precipitation icing accretion on overhead power lines, IEEE TRANSACTIONS ON POWER DELIVERY 20 (2005) 1080-1087.

[23] L. P. Kang Q., Zhang D., T. I., Lattice boltzmann model for crystal growth from supersaturated solution., Geophysical Research Letters 31 (2004) L21604.

[24] G. Lu, D. J. DePaolo, Q. Kang, D. Zhang, Lattice boltzmann simulation of snow crystal growth in clouds, Journal of Geophysical Research 114 (2009) D07305.

[25] D. Sun, M. Zhu, S. Pan, D. Raabe, Lattice boltzmann modeling of dendritic growth in a forced melt convection, Acta Materialia 57 (6) (2009) $1755-1767$.

[26] H. Yin, S. Felicelli, L. Wang, Simulation of a dendritic microstructure with the lattice boltzmann and cellular automaton methods, Acta Materialia 59 (8) (2011) $3124-3136$.

[27] L. Chen, Q. Kang, Y.-L. He, W.-Q. Tao, Mesoscopic study of the effects of gel concentration and materials on the formation of precipitation patterns, Langmuir 28 (32) (2012) 11745-11754. 
[28] L. Chen, Q. Kang, B. A. Robinson, Y.-L. He, W.-Q. Tao, Pore-scale modeling of multiphase reactive transport with phase transitions and dissolution-precipitation processes in closed systems, Physical Review E 87 (4) (2013) 043306.

[29] E. Maisse, J. Pousin, Diffusion and dissolution/precipitation in an open porous reactive medium, Journal of Computational and Applied Mathematics 82 (1997) 279.

[30] J. S. Chang, R. A. Brost, I. S. A. Isaksen, S. Madronich, P. Middleton, W. R. Stockwell, C. J. Walcek, A three-dimensional eulerian acid deposition model: Physical concepts and formulation, Journal of Geophysical Research 92 (1987) 14,681-14,700.

[31] X. Z, M. P., Phase-field modeling of solute precipitation and dissolution., J Chem Phys 129 (2008) 014705.

[32] M. Huneault, C. Langheit, J. Caron, Combined models for glaze ice accretion and de-icing of current-carring electrical conductors, IEEE TRANSACTIONS ON POWER DELIVERY 20 (2005) 1611-1616.

[33] L. Makkonen, Modeling power line icing in freezing precipitation, Atmospheric research 46 (1998) 131-142.

[34] M. Epstein, F. B. Cheung, Complex freezing-melting interfaces in fluid flow, Ann. Rev. Fluid Mech. 15 (1983) 293-319.

[35] V. I. Khvorostyanov, J. A. Curry, Thermodynamic theory of freezing and melting of water and aqueous solutions, J. Phys. Chem. A 108 (2004) 11073-11085.

[36] E. P. Lozowski, J. R. Stallabrass, P. F. Hearty, The icing of an unheated, nonrotating cylinder. part i: A simulation model, Journal of Climate and Applied Meteorology 22 (1983) 2053-2062.

[37] E. Javierre, C. Vuik, F. J. Vermolen, A. Segal, A level set method for three dimensional vector Stefan problems: Dissolution of stoichiometric particles in multi-component alloys, J. Comput. Phys. 224 (2007) 222240. 
[38] L. Tan, N. Zabaras, A level set simulation of dendritic solidification with combined features of front-tracking and fixed-domain methods, J. Comput. Phys. 211 (2006) 36-63.

[39] S. T. D. Tartakovsky A. M., Meakin P., W. R. M. E., Simulation of reactive transport and precipitation with smoothed particle hydrodynamics., Journal of Computational Physics 222 (2007) 654-672.

[40] N. Al-Rawahi, G. Tryggvason, Numerical simulation of dentritic solidification with convection: Three-dimensional flow, J. Comput. Phys. 194 (2004) 677-696.

[41] E. Widjaja, M. T. Harris, Particle deposition study during sessile drop evaporation, AIChE Journal 54 (2008) 2250-2260.

[42] J. Glimm, J. W. Grove, X.-L. Li, K.-M. Shyue, Q. Zhang, Y. Zeng, Three dimensional front tracking, SIAM J. Sci. Comp. 19 (1998) 703-727.

[43] B. Fix, J. Glimm, R. Kaufman, X. L. Li, L. L. Wu, Frontier and application to fluid instability study, verification and validation of frontier code and application to fluid interfacial instabilities, Physica ScriptaProceedings of World Conference on Turbulence Mixing and Beyond.

[44] F. Furtado, J. Glimm, J. W. Grove, X.-L. Li, W. B. Lindquist, R. Menikoff, D. H. Sharp, Q. Zhang, Front tracking and the interaction of nonlinear hyperbolic waves, Vol. 43, Springer-Verlag, New York, 1989, pp. 99-111, proceedings of the US-ROC Joint Symposium.

[45] X. Jiao, H. Zha, Consistent computation of first- and second-order differential quantities for surface meshes, in: Proceedings of the ACM Solid and Physical Modeling Symposium, 2008, pp. 159-170.

[46] D. Wang, B. L. Clark, X. Jiao, An analysis and comparison of parameterization-based computation of differential quantities for discrete surfaces, Comput. Aid. Geom. Des. 26 (2009) 510-527.

[47] X. Li, J. Glimm, X. Jiao, C. Peyser, Y. Zhao, Study of crystal growth and solute precipitation through front tracking method, Acta Mathematica Scientia 20 (2010) 377-390. 
[48] J. B. Bell, P. Colella, H. M. Glaz, A second-order projection method for the incompressible navier-stokes equations, Journal of Computational Physics 85 (1989) 257-283.

[49] G. Jiang, C.-W. Shu, Efficient implementation of weighted ENO schemes, J. Comput. Phys. 126 (1996) 202-228.

[50] V. Holten, C. E. Bertrand, M. A. Anisimov, J. V. Sengers, Thermodynamics of supercooled water, THE JOURNAL OF CHEMICAL PHYSICS 136 (2012) 094507.

[51] M. L. V. Ramires, C. A. N. de Castro, Y. Nagasaka, A. Nagashima, M. J. Assael, W. A. Wakeham, Standard reference data for the thermal conductivity of water, Journal of Physical and Chemical Refenrence Data 24 (1995) 1377-1381.

[52] I.-L. Chern, Y.-C. Shu, A coupling interface method for elliptic interface problems, Journal of Computational Physics 225 (2007) 2138-2174.

[53] N. L. Miles, J. Verlinde, E. E. Clothiaux, Cloud droplet size distributions in low-level stratiform clouds, Journal of the Atmospheric Sciences 57 (2000) 295-311. 